\title{
Existence of martingale solutions and the incompressible limit for stochastic compressible flows on the whole space
}

\author{
Prince Romeo Mensah $^{1}$ (D)
}

Received: 14 December 2016 / Accepted: 25 March 2017 / Published online: 11 May 2017

(C) The Author(s) 2017. This article is an open access publication

\begin{abstract}
We give an existence and asymptotic result for the so-called finite energy weak martingale solution of the compressible isentropic Navier-Stokes system driven by some random force in the whole spatial region. In particular, given a general nonlinear multiplicative noise, we establish the convergence to the incompressible system as the Mach number, representing the ratio between the average flow velocity and the speed of sound, approaches zero.
\end{abstract}

Keywords Isentropic flows · Stochastic compressible fluid · Navier-Stokes · Mach number · Martingale solution

Mathematics Subject Classification $35 \mathrm{R} 60 \cdot 35 \mathrm{Q} 35 \cdot 76 \mathrm{~N} 10 \cdot 76 \mathrm{M} 45$

\section{Introduction}

In continuum mechanics, the motion of an isentropic compressible fluid is described by the density $\varrho=\varrho(t, x)$ and velocity $\mathbf{u}=\mathbf{u}(t, x)$ in a physical domain in $\mathbb{R}^{3}$ satisfying the mass and momentum balance equations given, respectively, by

$$
\begin{aligned}
\partial_{t} \varrho+\operatorname{div}(\varrho \mathbf{u}) & =0 \\
\partial_{t}(\varrho \mathbf{u})+\operatorname{div}(\varrho \mathbf{u} \otimes \mathbf{u}) & =\operatorname{div} \mathbb{T}+\varrho \mathbf{f} .
\end{aligned}
$$

Here $\mathbf{f}$ is some external force and $\mathbb{T}$ the stress tensor. By Stokes' law, $\mathbb{T}$ satisfies $\mathbb{T}=\mathbb{S}-p \mathbb{I}$ where $p=p(\varrho)$ is the pressure and $\mathbb{S}=\mathbb{S}(\nabla \mathbf{u})$ the viscous stress tensor. In following Newton's law of viscosity, we assume that $\mathbb{S}$ satisfies

$$
\mathbb{S}=v\left(\nabla \mathbf{u}+\nabla^{T} \mathbf{u}\right)+\lambda \operatorname{div} \mathbf{u} \mathbb{I}
$$

\section{Prince Romeo Mensah}

pm27@hw.ac.uk

1 Department of Mathematics, Heriot-Watt University, Edinburgh EH14 4AS, UK 
with viscosity coefficients satisfying $v>0, \lambda+\frac{2}{3} v \geq 0$. For the pressure, we suppose the $\gamma$-law

$$
p=\frac{1}{\mathrm{Ma}^{2}} \varrho^{\gamma}
$$

where Ma $>0$ is the Mach number and $\gamma>\frac{3}{2}$ the adiabatic exponent. In order to study the existence of solutions to system (1), it has to be complemented by initial and boundary conditions (very common are periodic boundary conditions, no-slip boundary conditions and the whole space). The existence of weak solutions to (1) has been shown in the fundamental book by Lions [23] and extended to physical reasonable situations by Feireisl [11,15], giving a compressible analogue of the pioneering work by Leray [22] on the incompressible case. These results involve the concept of weak solutions where derivatives have to be understood in the sense of distributions. This concept has since become an integral technique in the study of nonlinear PDE's.

In recent years, there has been an increasing interest in random influences on fluid motions. It can take into account, for example, physical, empirical or numerical uncertainties and is commonly used to model turbulence in the fluid motion.

As far as we know, the first result on the existence of solution to the stochastic compressible system is due to [34]. This was done in 1-D and later for a special periodic 2-D case in [33]. The latter mostly relied on existence arguments developed in [35]. In [13], a semideterministic approach based on results on multi-valued functions is used and follows in line with the incompressible analogue shown in [1]. A fully stochastic theory has been developed in [5]. The existence of martingale solutions has been shown in the case of periodic boundary conditions. This has been extended to Dirichlet boundary conditions in [32].

Compared to the stochastic compressible model, the incompressible system has been studied more intensively. It first appeared in the seminal paper by Bensoussan and Temam [1] which is based on a semi-deterministic approach. Later, the concept of a martingale solution of this system was then introduced by Flandoli and Gatarek [16]. For a recent survey on the stochastic incompressible Navier-Stokes equations, we refer the reader to [30] or to [29] for the general survey including deterministic results.

The aim of this paper is to look at the situation on the whole space $\mathbb{R}^{3}$. This is particularly important for various applications and especially for those in which the comparative size of the fluids domain far exceeds the speed of sound accompanying the fluid. See [14] for more details. Difficulties arise due to the lack of certain compactness tools which are available in the case of bounded domains. We shall study the system

$$
\begin{gathered}
\mathrm{d} \varrho+\operatorname{div}(\varrho \mathbf{u}) \mathrm{d} t=0, \\
\mathrm{~d}(\varrho \mathbf{u})+[\operatorname{div}(\varrho \mathbf{u} \otimes \mathbf{u}-\mathbb{S}(\nabla \mathbf{u}))+\nabla p(\varrho)] \mathrm{d} t=\Phi(\varrho, \varrho \mathbf{u}) \mathrm{d} W,
\end{gathered}
$$

in $Q_{T}=(0, T) \times \mathbb{R}^{3}$. A prototype for the stochastic forcing term will be given by

$$
\Phi(\varrho, \varrho \mathbf{u}) \mathrm{d} W \approx \varrho \mathrm{d} W^{1}+\varrho \mathbf{u} \mathrm{d} W^{2}
$$

where $W^{1}$ and $W^{2}$ are a pair of independent cylindrical Wiener processes. We refer to Sect. 2 for the precise assumptions on the noise and its coefficients.

The first main result of the present paper is the existence of finite energy weak martingale solutions to (2). The precise statement is given in Theorem 1. We approximate the system on the whole space by a sequence of periodic problems (where the period tends to infinity). After showing a uniform a priori estimates, we use the stochastic compactness method based on the Jakubowski-Skorokhod representation theorem. In contrast to previous works, we 
adapt it to the situation on the whole space taking carefully into account the lack of compact embeddings. In order to pass to the limit in the nonlinear pressure term, we use properties of the effective viscous flux originally introduced by Lion [23] similar to [5].

A fundamental question in compressible fluid mechanics is the relation to the incompressible model. If the Mach number is small, the fluid should behave asymptotically like an incompressible one, provided velocity and viscosity are small, and we are looking at large time scales, see [21]. The problem has been studied rigorously in the deterministic case in [24-26], as a singular limit problem. A major problem to overcome is the rapid oscillation of acoustic waves due to the lack of compactness. A stochastic counterpart of this theory has very recently been established in [4]. The limit $\varepsilon$ of the system

$$
\begin{gathered}
\mathrm{d} \varrho_{\varepsilon}+\operatorname{div}\left(\varrho_{\varepsilon} \mathbf{u}_{\varepsilon}\right) \mathrm{d} t=0, \\
\mathrm{~d}\left(\varrho_{\varepsilon} \mathbf{u}_{\varepsilon}\right)+\left[\operatorname{div}\left(\varrho_{\varepsilon} \mathbf{u}_{\varepsilon} \otimes \mathbf{u}_{\varepsilon}-\mathbb{S}\left(\nabla \mathbf{u}_{\varepsilon}\right)\right)+\nabla \frac{\varrho_{\varepsilon}^{\gamma}}{\varepsilon^{2}}\right] \mathrm{d} t=\Phi\left(\varrho_{\varepsilon}, \varrho_{\varepsilon} \mathbf{u}_{\varepsilon}\right) \mathrm{d} W
\end{gathered}
$$

has been analyzed under periodic boundary conditions. Given a sequence of the so-called finite energy weak martingale solution for (4) (see next section for definition) where $\varepsilon \in(0,1)$, its limit (as $\varepsilon \rightarrow 0$ ) is indeed a weak martingale solution to the following incompressible system:

$$
\begin{gathered}
\operatorname{div}(\mathbf{u})=0 \\
\mathrm{~d}(\mathbf{u})+[\operatorname{div}(\mathbf{u} \otimes \mathbf{u})-v \Delta \mathbf{u}+\nabla \tilde{p}] \mathrm{d} t=\mathcal{P} \Phi(1, \mathbf{u}) \mathrm{d} W .
\end{gathered}
$$

Here $\tilde{p}$ is the associated pressure and $\mathcal{P}$ is the Helmholtz projection onto the space of solenoidal vector fields.

A major drawback in the approach in [4] is that the noise coefficient $\Phi(\varrho, \varrho \mathbf{u})$ has to be linear in the momentum $\varrho \mathbf{u}$. This is due to the aforementioned lack of compactness of momentum when $\varepsilon$ passes to zero. This cannot even be improved in the deterministic case. The situation on the whole space, however, is much better as a consequence of dispersive estimates for the acoustic wave equations, see Proposition 8. We apply them to the stochastic wave equation and hence are able to prove strong convergence of the momentum, see Lemma 10. Based on this, we are able to prove the convergence of (4) to (5) under much more general assumptions on the noise coefficients. See Theorem 2 for details.

In Sect. 2, we state the required assumptions satisfied by the various quantities used in this paper, as well as some useful function space estimates. We define the concept of a solution, state the required boundary condition applicable in our setting and finally state the main results.

In Sect. 3, we are concerned with the proof of Theorem 1, giving existence of martingale solutions on the whole space. Based on this result, we devote Sect. 4 to the proof of Theorem 2, the low-Mach number limit on the whole space.

\section{Preliminaries}

Throughout this paper, the spatial dimension is $N=3$ and we assume that $\left(\Omega, \mathscr{F},\left(\mathscr{F}_{t}\right)_{t \geq 0}, \mathbb{P}\right)$ is a stochastic basis with a complete right-continuous filtration, $W$ is a $(\mathscr{F} t)$-cylindrical Wiener process, that is, there exists a family of mutually independent real-valued Brownian motions $\left(\beta_{k}\right)_{k \in \mathbb{N}}$ and orthonormal basis $\left(e_{k}\right)_{k \in \mathbb{N}}$ of a separable Hilbert space $\mathfrak{U}$ such that 


$$
W(t)=\sum_{k \in \mathbb{N}} \beta_{k}(t) e_{k}, \quad t \in[0, T]
$$

We also assume that $\varrho \in L_{\mathrm{loc}}^{\gamma}\left(\mathbb{R}^{3}\right), \varrho \geq 0$, and $\mathbf{u} \in L_{\mathrm{loc}}^{2}\left(\mathbb{R}^{3}\right)$ so that $\sqrt{\varrho} \mathbf{u} \in L_{\mathrm{loc}}^{2}\left(\mathbb{R}^{3}\right)$.

Now let set $\mathbf{q}=\varrho \mathbf{u}$ a and assume that there exists a compact set $\mathcal{K} \subset \mathbb{R}^{3}$ and some functions $g_{k}: \mathbb{R}^{3} \times \mathbb{R} \times \mathbb{R}^{3} \rightarrow \mathbb{R}^{3}$ such that

$$
g_{k} \in C_{0}^{1}(\mathcal{K}), \quad \text { for any } k \in \mathbb{N},
$$

and in addition, satisfies the following growth conditions:

$$
\sum_{k \in \mathbb{N}}\left|g_{k}(x, \varrho, \mathbf{q})\right|^{2} \leq c\left(\varrho^{2}+|\mathbf{q}|^{2}\right), \quad \sum_{k \in \mathbb{N}}\left|\nabla_{\varrho, \mathbf{q}} g_{k}(x, \varrho, \mathbf{q})\right|^{2} \leq c .
$$

Then if we define the map $\Phi(\varrho, \varrho \mathbf{u}): \mathfrak{U} \rightarrow L^{1}(\mathcal{K})$ by $\Phi(\varrho, \varrho \mathbf{u}) e_{k}=g_{k}(\cdot, \varrho(\cdot), \varrho \mathbf{u}(\cdot))$, we can use the embedding $L^{1}(\mathcal{K}) \hookrightarrow W^{-l, 2}(\mathcal{K})$ where $l>\frac{3}{2}$, to show that $\|\Phi(\varrho, \varrho \mathbf{u})\|_{L_{2}\left(\mathfrak{U} ; W^{-l, 2}(\mathcal{K})\right)}^{2}$ is uniformly bounded provided $\varrho \in L_{\text {loc }}^{\gamma}\left(\mathbb{R}^{3}\right)$ and $\sqrt{\varrho} \mathbf{u} \in L_{\text {loc }}^{2}\left(\mathbb{R}^{3}\right)$. See [5, Eq. 2.3]. As such, the stochastic integral $\int_{0}^{\cdot} \Phi(\varrho, \varrho \mathbf{u}) \mathrm{d} W$ is a well-defined $\left(\mathscr{F}_{t}\right)$-martingale taking value in $W_{\text {loc }}^{-l, 2}\left(\mathbb{R}^{3}\right)$.

Lastly, we define the auxiliary space $\mathfrak{U}_{0} \supset \mathfrak{U}$ via

$$
\mathfrak{U}_{0}=\left\{\mathbf{u}=\sum_{k \geq 1} c_{k} e_{k} ; \quad \sum_{k \geq 1} \frac{c_{k}^{2}}{k^{2}}<\infty\right\}
$$

and endow it with the norm

$$
\|\mathbf{u}\|_{\mathfrak{U}_{0}}^{2}=\sum_{k \in \mathbb{N}} \frac{c_{k}^{2}}{k^{2}}, \quad \mathbf{u}=\sum_{k \in \mathbb{N}} c_{k} e_{k} .
$$

Then it can be shown that $W$ has $\mathbb{P}$-a.s. $C\left([0, T] ; \mathfrak{U}_{0}\right)$ sample paths with the Hilbert-Schmidt embedding $\mathfrak{U} \hookrightarrow \mathfrak{U}_{0}$. See [7].

\subsection{Sobolev inequalities for the homogeneous Sobolev space}

As we shall see shortly, the compactness techniques used in this paper involves certain estimates whose constants must necessarily be independent of the size of the domain. We therefore require the homogeneous Sobolev space

$$
D^{1, q}(\mathcal{O})= \begin{cases}\mathbf{u} \in \mathcal{D}^{\prime}(\mathcal{O}): \mathbf{u} \in L^{\frac{3 q}{3-q}}(\mathcal{O}), \nabla \mathbf{u} \in L^{q}(\mathcal{O}) & \text { if } 1 \leq q<3 \\ \mathbf{u}=\{\overline{\mathbf{u}}+c\}_{c \in \mathbb{R}}: \mathbf{u} \in L_{\mathrm{loc}}^{q}(\mathcal{O}), \nabla \mathbf{u} \in L^{q}(\mathcal{O}) & \text { if } q \geq 3\end{cases}
$$

which gives such Sobolev-type estimates. Here $\mathcal{O}$ is an exterior or an unbounded domain, for example $\mathcal{O}=\mathbb{R}^{3}$. In particular, given a function $\mathbf{u} \in D^{1, q}(\mathcal{O})$, we have that for any $1 \leq q<3$,

$$
\|\mathbf{u}\|_{L^{\frac{3 q}{3-q}(\mathcal{O})}} \leq c_{q}\|\nabla \mathbf{u}\|_{L^{q}(\mathcal{O})}
$$

See [17, Chapter II] for more details. Note that the constant above is independent of the size of $\mathcal{O}$, unlike in the case of the usual Sobolev-Poincáre's inequality.

To continue, let us define the concept of a solution used in this paper. 
Definition 1 If $\Lambda$ is a Borel probability measure on $L^{\gamma}\left(\mathbb{R}^{3}\right) \times L^{\frac{2 \gamma}{\gamma+1}}\left(\mathbb{R}^{3}\right)$, then we say that

$$
[(\Omega, \mathscr{F},(\mathscr{F} t), \mathbb{P}) ; \varrho, \mathbf{u}, W]
$$

is a finite energy weak martingale solution of Eq. (4) with initial law $\Lambda$ provided:

1. $\left(\Omega, \mathscr{F},\left(\mathscr{F}_{t}\right), \mathbb{P}\right)$ is a stochastic basis with a complete right-continuous filtration,

2. $W$ is a $\left(\mathscr{F}_{t}\right)$-cylindrical Wiener process,

3. the density $\varrho$ satisfies $\varrho \geq 0, t \rightarrow\langle\varrho(t, \cdot), \phi\rangle \in C[0, T]$ for any $\phi \in C_{c}^{\infty}\left(\mathbb{R}^{3}\right) \mathbb{P}-$ a.s., the function $t \mapsto\langle\varrho(t, \cdot), \phi\rangle$ is progressively measurable, and

$$
\mathbb{E}\left[\sup _{t \in[0, T]}\|\varrho(t, \cdot)\|_{L^{\gamma}(\mathcal{K})}^{p}\right]<\infty \text { for all } 1 \leq p<\infty,
$$

for all $\mathcal{K} \subset \mathbb{R}^{3}$ with $\mathcal{K}$ compact,

4. the momentum $\varrho \mathbf{u}$ satisfies $t \rightarrow\langle\varrho \mathbf{u}, \phi\rangle \in C[0, T]$ for any $\phi \in C_{c}^{\infty}\left(\mathbb{R}^{3}\right) \mathbb{P}-$ a.s., the function $t \mapsto\langle\varrho \mathbf{u}, \phi\rangle$ is progressively measurable, and for all $1 \leq p<\infty$

$$
\mathbb{E}\left[\sup _{t \in[0, T]}\|\sqrt{\varrho} \mathbf{u}\|_{L^{2}(\mathcal{K})}^{p}\right]<\infty, \quad \mathbb{E}\left[\sup _{t \in[0, T]}\|\varrho \mathbf{u}\|_{L^{\frac{2 \gamma}{\gamma+1}}(\mathcal{K})}^{p}\right]<\infty,
$$

for all $\mathcal{K} \subset \mathbb{R}^{3}, \mathcal{K}$ compact,

5. the velocity field $\mathbf{u}$ is $\left(\mathscr{F}_{t}\right)$-adapted, $\mathbf{u} \in L^{p}\left(\Omega ; L^{2}\left(0, T ; W_{\text {loc }}^{1,2}\left(\mathbb{R}^{3}\right)\right)\right)$ and,

$$
\mathbb{E}\left[\left(\int_{0}^{T}\|\mathbf{u}\|_{W^{1,2}(\mathcal{K})}^{2} \mathrm{~d} t\right)^{p}\right]<\infty \text { for all } 1 \leq p<\infty,
$$

for all $\mathcal{K} \subset \mathbb{R}^{3}, \mathcal{K}$ compact,

6. $\Lambda=\mathbb{P} \circ(\varrho(0), \varrho \mathbf{u}(0))^{-1}$,

7. for all $\psi \in C_{c}^{\infty}\left(\mathbb{R}^{3}\right)$ and $\phi \in C_{c}^{\infty}\left(\mathbb{R}^{3}\right)$ and all $t \in[0, T]$, it holds $\mathbb{P}$-a.s.

$$
\begin{aligned}
\langle\varrho(t), \psi\rangle= & \langle\varrho(0), \psi\rangle+\int_{0}^{t}\langle\varrho \mathbf{u}, \nabla \psi\rangle \mathrm{d} s, \\
\langle\varrho \mathbf{u}(t), \phi\rangle= & \langle\varrho \mathbf{u}(0), \phi\rangle+\int_{0}^{t}\langle\varrho \mathbf{u} \otimes \mathbf{u}, \nabla \phi\rangle \mathrm{d} s-v \int_{0}^{t}\langle\nabla \mathbf{u}, \nabla \phi\rangle \mathrm{d} s \\
& -(\lambda+v) \int_{0}^{t}\langle\operatorname{div} \mathbf{u}, \operatorname{div} \phi\rangle \mathrm{d} s+\frac{1}{\mathrm{Ma}^{2}} \int_{0}^{t}\left\langle\varrho^{\gamma}, \operatorname{div} \phi\right\rangle \mathrm{d} s \\
& +\int_{0}^{t}\langle\Phi(\varrho, \varrho \mathbf{u}) \mathrm{d} W, \phi\rangle,
\end{aligned}
$$

8. for any $1 \leq p<\infty$, the energy estimate holds

$$
\begin{aligned}
& \mathbb{E}\left[\sup _{t \in[0, T]} \int_{\mathbb{R}^{3}}\left(\frac{\varrho(t)|\mathbf{u}(t)|^{2}}{2}+H(\varrho(t))\right) \mathrm{d} x\right]^{p}+\mathbb{E}\left[\int_{Q_{T}} \mathbb{S}(\nabla \mathbf{u}): \nabla \mathbf{u} \mathrm{d} x \mathrm{~d} s\right]^{p} \\
& \leq c_{p}\left(1+\mathbb{E}\left[\int_{\mathbb{R}^{3}}\left(\frac{\left|\mathbf{q}_{0}\right|^{2}}{2 \varrho_{0}}+H(\varrho(0, \cdot))\right) \mathrm{d} x\right]^{p}\right)
\end{aligned}
$$


where $Q_{T}:=(0, T) \times \mathbb{R}^{3}$ and where

$$
H(\varrho)=\frac{a}{\gamma-1}\left(\varrho^{\gamma}-\gamma \bar{\varrho}^{\gamma-1}(\varrho-\bar{\varrho})-\bar{\varrho}^{\gamma}\right) .
$$

is the pressure potential for constants $a, \bar{\varrho}>0$.

9. In addition, (2) holds in the renormalized sense. That is, for any $\phi \in \mathcal{D}^{\prime}\left(\mathbb{R}^{3}\right)$ and $b(\varrho) \in$ $C^{0}[0, \infty) \cap C^{1}(0, \infty)$ such that $\left|b^{\prime}(t)\right| \leq c t^{-\lambda_{0}}, t \in(0,1], \lambda_{0}<1$ and $\left|b^{\prime}(t)\right| \leq c t^{\lambda_{1}}$, $t \geq 1$ where $c>0$ and $-1 \leq \lambda_{1}<\infty$, we have that

$$
\mathrm{d}\langle b(\varrho), \phi\rangle=\langle b(\varrho) \mathbf{u}, \nabla \phi\rangle \mathrm{d} t-\left\langle\left(b(\varrho)-b^{\prime}(\varrho) \varrho\right) \operatorname{div} \mathbf{u}, \phi\right\rangle \mathrm{d} t .
$$

Remark 1 The definition above also holds for functions defined on the periodic space $\mathbb{T}_{L}^{3}=$ $\left(\left.[-L, L]\right|_{\{-L, L\}}\right)^{3}=(\mathbb{R} \mid 2 L \mathbb{Z})^{3}$ for any $L \geq 1$, rather than on the whole space $\mathbb{R}^{3}$. In that case, it even suffices to consider just smooth test functions which are not necessarily compactly supported. See for example [3-5].

Definition 2 If $\Lambda$ is a Borel probability measure on $L_{d i v}^{2}\left(\mathbb{R}^{3}\right)$, then we say that $\left[\left(\Omega, \mathscr{F},\left(\mathscr{F}_{t}\right)\right.\right.$, $\mathbb{P}), \mathbf{u}, W]$ is a weak martingale solution of Eq. (5) with initial law $\Lambda$ provided:

1. $\left(\Omega, \mathscr{F},\left(\mathscr{F}_{t}\right), \mathbb{P}\right)$ is a stochastic basis with a complete right-continuous filtration,

2. $W$ is a $\left(\mathscr{F}_{t}\right)$-cylindrical Wiener process,

3. $\mathbf{u}$ is $\left(\mathscr{F}_{t}\right)$-adapted, $\mathbf{u} \in C_{w}\left([0, T] ; L_{\text {div }}^{2}\left(\mathbb{R}^{3}\right)\right) \cap L^{2}\left(0, T ; W_{\text {div }}^{1,2}\left(\mathbb{R}^{3}\right)\right) \mathbb{P}-$ a.s. and,

$$
\mathbb{E}\left[\sup _{(0, T)}\|\mathbf{u}\|_{L^{2}\left(\mathbb{R}^{3}\right)}^{2}\right]^{p}+\mathbb{E}\left[\left(\int_{0}^{T}\|\mathbf{u}\|_{W^{1,2}\left(\mathbb{R}^{3}\right)}^{p} \mathrm{~d} t\right)^{p}\right]<\infty \quad \text { for all } 1 \leq p<\infty,
$$

4. $\Lambda=\mathbb{P} \circ(\mathbf{u}(0))^{-1}$,

5. for all $\phi \in C_{c, \text { div }}^{\infty}\left(\mathbb{R}^{3}\right)$ and all $t \in[0, T]$, it holds $\mathbb{P}$-a.s.

$$
\langle\mathbf{u}(t), \phi\rangle=\langle\mathbf{u}(0), \phi\rangle+\int_{0}^{t}\langle\mathbf{u} \otimes \mathbf{u}, \nabla \phi\rangle \mathrm{d} s-v \int_{0}^{t}\langle\nabla \mathbf{u}, \nabla \phi\rangle \mathrm{d} s+\int_{0}^{t}\langle\mathcal{P} \Phi(1, \mathbf{u}) \mathrm{d} W, \phi\rangle,
$$

Existence of weak martingale solutions as defined in Definition 2 has been shown to exist under suitable growth conditions on the noise term. We refer the reader to [27], albeit stated in the Stratonovich sense. A global-in-space existence result stated in the Itô form appears to be absent from the literatures although it is certainly expected. However, this is a by product of the singular limit problem that we study in this paper. See Theorem 2 below. For bounded domains, see for example, $[6,16]$.

\subsection{Prescribed boundary conditions}

Let assume that the right-hand side of the energy inequality (10) is finite. Then, we can deduce from (11) that

$$
\lim _{|x| \rightarrow \infty} \varrho(x)=\bar{\varrho}
$$

for some $\bar{\varrho}>0$. This is because if we apply Taylor's expansion around the constant $\bar{\varrho}$ for the function $f(\varrho)=\varrho^{\gamma}$, we can rewrite (11) as

$$
H(\varrho)=\frac{a \gamma z^{\gamma-2}}{2}(\varrho-\bar{\varrho})^{2}, \quad z \in[\varrho, \bar{\varrho}] \text { or } z \in[\bar{\varrho}, \varrho]
$$


and so the boundedness of the left-hand side of (10) means that the difference $\varrho-\bar{\varrho} \in$ $L^{p}\left(\Omega ; L^{\infty}\left(0, T ; L^{\min \{2, \gamma\}}\left(\mathbb{R}^{3}\right)\right)\right)$ when (14) is substituted into (10).

Furthermore, we also have that $\varrho|\mathbf{u}|^{2} \in L^{1}\left(\Omega ; L^{\infty}\left(0, T ; L^{1}\left(\mathbb{R}^{3}\right)\right)\right)$ and as such,

$$
\lim _{|x| \rightarrow \infty} \varrho(x)|\mathbf{u}(x)|^{2}=0 .
$$

By combining (13) and (15) (keeping in mind that $\bar{\varrho} \neq 0$ ), it is reasonable to impose the boundary condition

$$
\lim _{|x| \rightarrow \infty} \mathbf{u}(x)=0
$$

\subsection{Main results}

We now state the main results of this paper.

Theorem 1 Let $\gamma>\frac{3}{2}$ and let $\Lambda$ be a probability law on $L^{\gamma}\left(\mathbb{R}^{3}\right) \times L^{\frac{2 \gamma}{\gamma+1}}\left(\mathbb{R}^{3}\right)$ satisfying

$$
\begin{gathered}
\Lambda\left\{(\varrho, \mathbf{q}) \in L^{\gamma}\left(\mathbb{R}^{3}\right) \times L^{\frac{2 \gamma}{\gamma+1}}\left(\mathbb{R}^{3}\right): \varrho \geq 0, M_{1}^{\mathcal{K}} \leq \int_{\mathcal{K}} \varrho \mathrm{d} x \leq M_{2}^{\mathcal{K}},\left.\mathbf{q}\right|_{\{\varrho=0\}}=0,\right. \\
\left.\left|\frac{\varrho-1}{\varepsilon}\right| \leq M_{2}^{\mathcal{K}}\right\}=1, \int_{L_{x}^{\gamma} \times L_{x}^{\gamma+1}} \frac{2 \gamma}{2} \frac{|\mathbf{q}|^{2}}{\varrho}+H(\varrho) \|_{L_{x}^{1}}^{p} \mathrm{~d} \Lambda(\varrho, \mathbf{q}) \leq c_{p}<\infty,
\end{gathered}
$$

for all $0 \leq p<\infty$ and any compact set $\mathcal{K} \subset \mathbb{R}^{3}$ with constants $0<M_{1}^{\mathcal{K}}<M_{2}^{\mathcal{K}}$ which are independent of $\varepsilon \in(0,1)$. Also assume that (6) and (7) holds. Then there exists a finite energy weak martingale solution of (4) in the sense of Definition 1, with initial law $\Lambda$.

Remark 2 The assumption $\left|\frac{\varrho-1}{\varepsilon}\right| \leq M_{2}^{\mathcal{K}}$ given in the law above is not restrictive and can actually be dropped. However, it is needed in the proof of Theorem 2.

Theorem 2 Let $\Lambda$ be a given Borel probability measure on $L^{2}\left(\mathbb{R}^{3}\right)$. For $\varepsilon \in(0,1)$, we let $\Lambda_{\varepsilon}$, be a Borel probability measure on $L^{\gamma}\left(\mathbb{R}^{3}\right) \times L^{\frac{2 \gamma}{\gamma+1}}\left(\mathbb{R}^{3}\right)$ where $\gamma>3 / 2$ is such that the initial law in Theorem 1 holds and where the marginal law of $\Lambda_{\varepsilon}$ corresponding to the second component converges to $\Lambda$ weakly in the sense of measures on $L^{\frac{2 \gamma}{\gamma+1}}\left(\mathbb{R}^{3}\right)$. Also assume that (6) and (7) holds. If $\left[\left(\Omega^{\varepsilon}, \mathscr{F}^{\varepsilon},\left(\mathscr{F}_{t}^{\varepsilon}\right), \mathbb{P}^{\varepsilon}\right) ; \varrho_{\varepsilon}, \mathbf{u}_{\varepsilon}, W_{\varepsilon}\right]$ is a finite energy weak martingale solution of (4) with initial law $\Lambda_{\varepsilon}$, then

$$
\begin{aligned}
\left(\varrho_{\varepsilon}-1\right) & \rightarrow 0 \text { in law in } L^{\infty}\left(0, T ; L^{\min \{2, \gamma\}}\left(\mathbb{R}^{3}\right)\right) \\
\mathbf{u}_{\varepsilon} & \rightarrow \mathbf{u} \text { in law in }\left(L^{2}\left(0, T ; W_{\mathrm{loc}}^{1,2}\left(\mathbb{R}^{3}\right)\right), w\right) \\
\varrho_{\varepsilon} \mathbf{u}_{\varepsilon} & \rightarrow \mathbf{u} \text { in law in } L^{2}\left(0, T ; L_{\mathrm{loc}}^{r}\left(\mathbb{R}^{3}\right)\right)
\end{aligned}
$$

where $\mathbf{u}$ is a weak martingale solution of (5) in the sense of Definition 2 with the initial law $\Lambda$ and $r \in\left(\frac{3}{2}, 6\right)$.

\section{Proof of Theorem 1}

Let $\varrho_{L}$ and $\mathbf{u}_{L}$ be some density and velocity fields defined $\mathrm{d} \mathbb{P} \times \mathrm{d} t$ a.e. $(\omega, t) \in \Omega \times[0, T]$ on the space $\mathbb{T}_{L}^{3}$ such that $\varrho_{L}$ and $\mathbf{u}_{L}$ satisfies the so-called dissipative estimate; existence of which is shown in [3, Eq. 3.2] for the particular choice of $L=1$. 
We observe that [3, Eq. 3.2] is translation invariant and as such, holds true for any fixed $L \geq 1$. Also, the inequality is preserved if we replace $H_{\delta}(\varrho)$ by $H(\varrho)$. As such if we consider $\psi=\chi_{[0, t]}$, then we obtain the inequality:

$$
\begin{aligned}
\int_{0}^{t} & \int_{\mathbb{T}_{L}^{3}} \mathbb{S}\left(\nabla \mathbf{u}_{L}\right): \nabla \mathbf{u}_{L} \mathrm{~d} x \mathrm{~d} s+\int_{\mathbb{T}_{L}^{3}}\left[\frac{\varrho_{L}(t)\left|\mathbf{u}_{L}(t)\right|^{2}}{2}+H\left(\varrho_{L}(t)\right)\right] \mathrm{d} x \\
\leq & \int_{\mathbb{T}_{L}^{3}}\left[\frac{\left|\left(\varrho_{L} \mathbf{u}_{L}\right)(0)\right|^{2}}{2 \varrho_{L}(0)}+H\left(\varrho_{L}(0)\right)\right] \mathrm{d} x+\int_{0}^{t} \int_{\mathbb{T}_{L}^{3}} \mathbf{u}_{L} \cdot \Phi\left(\varrho_{L}, \varrho_{L} \mathbf{u}_{L}\right) \mathrm{d} x \mathrm{~d} W \\
& +\int_{0}^{t} \int_{\mathbb{T}_{L}^{3}} \sum_{k \in \mathbb{N}} \frac{\left|g_{k}\left(\varrho_{L}, \varrho_{L} \mathbf{u}_{L}\right)\right|^{2}}{2 \varrho_{L}} \mathrm{~d} x \mathrm{~d} s
\end{aligned}
$$

However, due to (6), there is a compact set $\mathcal{K} \subset \mathbb{R}^{3}$ such that for any $1 \leq p<\infty$, we have that

$$
\begin{aligned}
\mathbb{E} \sup _{t \in[0, T]} \mid & \left.\int_{0}^{t} \int_{\mathbb{T}_{L}^{3}} \sum_{k \in \mathbb{N}} \frac{\left|g_{k}\left(\varrho_{L}, \varrho_{L} \mathbf{u}_{L}\right)\right|^{2}}{2 \varrho_{L}} \mathrm{~d} x \mathrm{~d} s\right|^{p} \\
& \leq \mathbb{E}\left(\int_{0}^{T} \int_{\mathbb{T}_{L}^{3}} \sum_{k \in \mathbb{N}} \frac{\left|g_{k}\left(\varrho_{L}, \varrho_{L} \mathbf{u}_{L}\right)\right|^{2}}{2 \varrho_{L}} \mathrm{~d} x \mathrm{~d} s\right)^{p} \\
& \leq c \mathbb{E}\left(\int_{0}^{T} \int_{\mathcal{K}} \varrho_{L}^{-1}\left(\varrho_{L}^{2}+\left|\varrho_{L} \mathbf{u}_{L}\right|^{2}\right) \mathrm{d} x \mathrm{~d} s\right)^{p} \\
& \leq c_{p} \mathbb{E} \int_{0}^{T}\left(\int_{\mathcal{K}}\left(1+\varrho_{L}^{\gamma}+\varrho_{L}\left|\mathbf{u}_{L}\right|^{2}\right) \mathrm{d} x\right)^{p} \mathrm{~d} s
\end{aligned}
$$

where $c_{p}$ is independent of both $k$ and $L$ and where we have used $\varrho \leq 1+\varrho^{\gamma}$.

Also, by use of the Burkholder-Davis-Gundy inequality, the Hölder's and Young's inequalities, we have that

$$
\begin{aligned}
& \mathbb{E} {\left[\sup _{t \in[0, T]}\left|\int_{0}^{t} \int_{\mathbb{T}_{L}^{3}} \mathbf{u}_{L} \cdot \Phi\left(\varrho_{L}, \mathbf{q}_{L}\right) \mathrm{d} x \mathrm{~d} W\right|\right]^{p} } \\
&= \mathbb{E}\left[\sup _{t \in[0, T]}\left|\int_{0}^{t} \sum_{k \in \mathbb{N}} \int_{\mathbb{T}_{L}^{3}} \mathbf{u}_{L} \cdot g_{k}\left(\varrho_{L}, \mathbf{q}_{L}\right) \mathrm{d} x \mathrm{~d} \beta_{k}\right|\right]^{p} \\
& \leq c_{p} \mathbb{E}\left[\int_{0}^{T} \sum_{k \in \mathbb{N}}\left(\int_{\mathbb{T}_{L}^{3}} \mathbf{u}_{L} \cdot g_{k}\left(\varrho_{L}, \mathbf{q}_{L}\right) \mathrm{d} x\right)^{2} \mathrm{~d} s\right]^{\frac{p}{2}} \\
& \leq c_{p} \mathbb{E}\left[\int_{0}^{T} \sum_{k \in \mathbb{N}}\left(\int_{\mathbb{T}_{L}^{3}}\left|\sqrt{\varrho_{L}} \mathbf{u}_{L}\right|^{2} \mathrm{~d} x\right)\left(\int_{\mathbb{T}_{L}^{3}}\left|\frac{g_{k}\left(\varrho_{L}, \mathbf{q}_{L}\right)}{\sqrt{\varrho_{L}}}\right|^{2} \mathrm{~d} x\right) \mathrm{d} s\right]^{\frac{p}{2}} \\
& \leq \epsilon \mathbb{E}\left(\sup _{t \in[0, T]} \int_{\mathbb{T}_{L}^{3}}\left|\sqrt{\varrho_{L}} \mathbf{u}_{L}\right|^{2} \mathrm{~d} x\right)^{p}+c_{p, \epsilon} \mathbb{E}_{0}^{T}\left(\int_{\mathcal{K}}\left(1+\varrho_{L}^{\gamma}+\varrho_{L}\left|\mathbf{u}_{L}\right|^{2}\right) \mathrm{d} x\right)^{p} \mathrm{~d} s
\end{aligned}
$$

for an arbitrarily small $\epsilon>0$. 
By taking the $p$ th-moment of the supremum in (17) and applying Gronwall's lemma, we obtain the inequality:

$$
\begin{gathered}
\mathbb{E}\left[\sup _{t \in[0, T]} \int_{\mathbb{T}_{L}^{3}}\left(\frac{\varrho_{L}\left|\mathbf{u}_{L}\right|^{2}}{2}+H\left(\varrho_{L}\right)\right) \mathrm{d} x\right]^{p}+\mathbb{E}\left[\int_{0}^{T} \int_{\mathbb{T}_{L}^{3}} \mathbb{S}\left(\nabla \mathbf{u}_{L}\right): \nabla \mathbf{u}_{L} \mathrm{~d} x \mathrm{~d} s\right]^{p} \\
\leq c_{p, \epsilon, \operatorname{vol}(\mathcal{K})}\left(1+\mathbb{E}\left[\int_{\mathbb{T}_{L}^{3}}\left[\frac{\left|\mathbf{q}_{L, 0}\right|^{2}}{2 \varrho_{L, 0}}+H\left(\varrho_{L}(0, \cdot)\right)\right] \mathrm{d} x\right]^{p}\right)
\end{gathered}
$$

where $c_{p, \epsilon, \operatorname{vol}(\mathcal{K})}$ is in particular, independent of $L$. Now by the assumptions on $\Lambda$, the right hand side of (18) is finite. As such, As such, we obtain the following uniform bounds in $L$ :

$$
\begin{aligned}
\sqrt{\varrho_{L}} \mathbf{u}_{L} & \in L^{p}\left(\Omega ; L^{\infty}\left(0, T ; L^{2}\left(\mathbb{T}_{L}^{3}\right)\right)\right), \\
\nabla u_{L} & \in L^{p}\left(\Omega ; L^{2}\left(0, T ; L^{2}\left(\mathbb{T}_{L}^{3}\right)\right)\right), \\
H\left(\varrho_{L}\right) & \in L^{p}\left(\Omega ; L^{\infty}\left(0, T ; L^{1}\left(\mathbb{T}_{L}^{3}\right)\right)\right), \\
\left(\varrho_{L}-\bar{\varrho}\right) & \in L^{p}\left(\Omega ; L^{\infty}\left(0, T ; L^{\min \{2, \gamma\}}\left(\mathbb{T}_{L}^{3}\right)\right)\right) .
\end{aligned}
$$

Note that the estimates in (19) are global but unfortunately, do not include all necessary quantities. In the following, we derive local estimates with respect to balls $B_{r}$ which will depend on the radius $r>0$. A consequence of $(19)_{3}$ is

$$
\varrho_{L} \in L^{p}\left(\Omega ; L^{\infty}\left(0, T ; L^{\gamma}\left(B_{r}\right)\right)\right)
$$

uniformly in $L$ (but depending on $r$ ). If $B_{r} \subset \mathbb{T}_{L}^{3}$, this follows in an obvious way from the definition of $H$. Otherwise we cover $B_{r} \subset \mathbb{R}^{3}$ by tori to which $\varrho_{L}$ is extended by means of periodicity. The number of necessary tori depends on $r$ but is independent of $L$. To see this, we notice that $\operatorname{since} \operatorname{vol}\left(B_{r}\right) \approx c(\pi) r^{3}$ and $\operatorname{vol}\left(\mathbb{T}_{L}^{3}\right) \approx c(\pi) L^{3}$, we will require $\mathcal{O}\left(\frac{r^{3}}{L^{3}}\right)$ number of tori to cover $B_{r}$. But since $L \geq 1$, we infact require $\mathcal{O}\left(r^{3}\right)$ (which is independent of $L$ ) number of such tori to cover $B_{r}$.

Remark 3 We get (20) be making it the subject in (11) and using (19) ${ }_{[3,4]}$. However, we only obtain the estimate locally in space because of the constant term $\bar{\varrho}$ in the pressure potential (11). This will blow up with the size of the torus if we try obtaining a global estimate.

We observe that non of the bounds in (19) directly controls the amplitude of $\mathbf{u}_{L}$. However using the Sobolev-Poincaré's inequality and $\gamma>\frac{3}{2}$, the following holds

$$
\begin{aligned}
& \left\|\varrho_{0}\right\|_{L^{1}\left(B_{r}\right)}\left|\left(\mathbf{u}_{L}\right)_{B_{r}}\right|=\left|\int_{B_{r}} \varrho\left(\mathbf{u}_{L}\right)_{B_{r}} \mathrm{~d} x\right| \\
& \quad \leq c \int_{B_{r}} \varrho\left|\left(\mathbf{u}_{L}\right)_{B_{r}}-\mathbf{u}_{L}\right| \mathrm{d} x+\int_{B_{r}} \varrho_{L}\left|\mathbf{u}_{L}\right| \mathrm{d} x \\
& \quad \leq c\left\|\varrho_{L}\right\|_{L^{\gamma}\left(B_{r}\right)}\left\|\left(\mathbf{u}_{L}\right)_{B_{r}}-\mathbf{u}_{L}\right\|_{L^{\gamma^{\prime}\left(B_{r}\right)}}+c\left\|\sqrt{\varrho_{L}}\right\|_{L^{2}\left(B_{r}\right)}\left\|\sqrt{\varrho_{L}} \mathbf{u}_{L}\right\|_{L^{2}\left(B_{r}\right)} \\
& \quad \leq c(r)\left\|\varrho_{L}\right\|_{L^{\gamma}\left(B_{r}\right)}\left\|\left(\mathbf{u}_{L}\right)_{B_{r}}-\mathbf{u}_{L}\right\|_{L^{6}\left(B_{r}\right)}+c\left\|\sqrt{\varrho_{L}}\right\|_{L^{2 \gamma}\left(B_{r}\right)}\left\|\sqrt{\varrho_{L}} \mathbf{u}_{L}\right\|_{L^{2}\left(B_{r}\right)} \\
& \quad \leq c(r)\left\|\varrho_{L}\right\|_{L^{\gamma}\left(B_{r}\right)}\left\|\nabla \mathbf{u}_{L}\right\|_{L^{2}\left(B_{r}\right)}+c \varrho_{\varrho_{L}}\left\|_{L^{\gamma}\left(B_{r}\right)}^{\frac{1}{2}}+c\right\| \varrho_{L}\left|\mathbf{u}_{L}\right|^{2} \|_{L^{1}\left(B_{r}\right)},
\end{aligned}
$$


and, consequently,

$$
\begin{aligned}
\left\|\varrho_{0}\right\|_{L^{1}\left(B_{r}\right)}^{2} \int_{0}^{\tau}\left|\left(\mathbf{u}_{L}\right)_{B_{r}}\right|^{2} \mathrm{~d} t \leq & c(r) \sup _{t \in[0, \tau]}\left\|\varrho_{L}\right\|_{L^{\gamma}\left(B_{r}\right)}^{2} \int_{0}^{\tau}\left\|\nabla \mathbf{u}_{L}\right\|_{L^{2}\left(B_{r}\right)}^{2} \mathrm{~d} t \\
& +c \tau \sup _{t \in(0, \tau)}\left(\left\|\varrho_{L}\right\|_{L^{1}\left(B_{r}\right)}^{2}+\left\|\varrho_{L}\left|\mathbf{u}_{L}\right|^{2}\right\|_{L^{1}\left(B_{r}\right)}^{2}\right) .
\end{aligned}
$$

In view of the bounds established in (19), (20) and the assumptions on the initial law, we can conclude that

$$
\mathbf{u}_{L} \in L^{p}\left(\Omega ; L^{2}\left(0, T ; W^{1,2}\left(B_{r}\right)\right)\right) .
$$

uniformly in $L$.

Furthermore, for $r>0$, we can use the (uniform in $L$ but not in $r$ ) continuous embedding $W^{1,2}\left(B_{r}\right) \hookrightarrow L^{6}\left(B_{r}\right)$ and Hölder's inequality, to get for $\mathrm{d} \mathbb{P} \times \mathrm{d} t$ a.e. $(\omega, t) \in \Omega \times[0, T]$,

$$
\begin{aligned}
\left\|\varrho_{L} \mathbf{u}_{L}\right\|_{L^{\frac{2 \gamma}{\gamma+1}\left(B_{r}\right)}} & \leq\left\|\sqrt{\varrho_{L}}\right\|_{L^{2 \gamma}\left(B_{r}\right)}\left\|\sqrt{\varrho_{L}} \mathbf{u}_{L}\right\|_{L^{2}\left(B_{r}\right)} \\
& =\left\|\varrho_{L}\right\|_{L^{\gamma}\left(B_{r}\right)}^{\frac{1}{2}}\left\|\sqrt{\varrho_{L}} \mathbf{u}_{L}\right\|_{L^{2}\left(B_{r}\right)},\left\|\varrho_{L} \mathbf{u}_{L} \otimes \mathbf{u}_{L}\right\|_{L^{\frac{6 \gamma}{4 \gamma+3}}\left(B_{r}\right)}, \\
& \leq\left\|\varrho_{L} \mathbf{u}_{L}\right\|_{L^{\frac{2 \gamma}{\gamma+1}\left(B_{r}\right)}}\left\|\mathbf{u}_{L}\right\|_{L^{6}\left(B_{r}\right)} .
\end{aligned}
$$

Since the radius of the ball above is chosen arbitrarily, we may conclude that

$$
\begin{array}{r}
\varrho_{L} \mathbf{u}_{L} \in L^{p}\left(\Omega ; L^{\infty}\left(0, T ; L^{\frac{2 \gamma}{\gamma+1}}\left(B_{r}\right)\right)\right), \\
\varrho_{L} \mathbf{u}_{L} \otimes \mathbf{u}_{L} \in L^{p}\left(\Omega ; L^{2}\left(0, T ; L^{\frac{6 \gamma}{4 \gamma+3}}\left(B_{r}\right)\right)\right),
\end{array}
$$

uniformly in $L$ for $r>0$ by using (19).

\subsection{Higher integrability of density}

For reasons that will be clear in the subsequent sections, it is essential to improve the regularity of density. We give this in the following lemma:

Lemma 1 Let $B_{r} \subset \mathbb{R}^{3}$ be a ball of radius $r>0$. Then for all $\Theta \leq \frac{2}{3} \gamma-1$, we have that

$$
\mathbb{E} \int_{0}^{T} \int_{B_{r}} a \varrho_{L}^{\gamma+\Theta} \mathrm{d} x \mathrm{~d} t \leq c
$$

where the constant $c$, is independent of $L$.

Proof If we set $B_{r, L}^{3}:=B_{r} \cap \mathbb{T}_{L}^{3}$, then it is enough to prove that

$$
\mathbb{E} \int_{0}^{T} \int_{B_{r, L}^{3}} a \varrho_{L}^{\gamma+\Theta} \mathrm{d} x \mathrm{~d} t \leq c
$$

independently of $L$. The general case then follows by covering $B_{r}$ by sets of the form $B \cap \mathbb{T}_{L}^{3}$ for a ball $B$. First notice that by combining (8) with the continuity property of the Bogovskir operator $\mathbb{B}\left(\varrho_{L}^{\Theta}\right)=\mathcal{B}\left[\varrho_{L}^{\Theta}-f \varrho_{L}^{\Theta} \mathrm{d} x\right]$, where $\mathcal{B}=\mathcal{B}_{B_{r, L}^{3}}$ is as defined in [9, Theorem 5.2] for the set $B_{r, L}^{3}$, we ensures that

$$
\left\|\mathbb{B}\left(\varrho_{L}^{\Theta}\right)\right\|_{L^{\frac{3 q}{3-q}}\left(B_{r, L}^{3}\right)} \leq c\left\|\varrho_{L}^{\Theta}\right\|_{L^{q}\left(B_{r, L}^{3}\right)}, \quad r>0
$$


holds uniformly in $L$ for $1 \leq q<3$.

Remark 4 Note that infact the set $B_{r, L}^{3}$ is a bounded John domain and hence satisfies the emanating chain condition with some constants $\sigma_{1}$ and $\sigma_{2}$ which are independent of the size of the torus. The fact that the constant $c$ in (26) is independent of $L$ therefore follows from the fact that the constant $c$ in [9, Theorem 5.2] only depends on $\sigma_{1}, \sigma_{2}$ and $q$ as well as the fact that $c_{q}$ is independent of $L$.

The idea now is to test the momentum equation with $\mathbb{B}\left(\varrho^{\Theta}\right)$. To do this, however, we first replace the map $\varrho \mapsto \varrho^{\Theta}$ with the function $b(\varrho) \in C_{c}^{1}(\mathbb{R})$ and apply Itó formula to the function $f(b, \mathbf{q})=\int_{B_{r . L}^{3}} \mathbf{q} \cdot \mathbb{B}(b(\varrho)) \mathrm{d} x$ where $\mathbb{B}(b(\varrho))=\mathbb{B}[b(\varrho)-f b(\varrho) \mathrm{d} x]$. Since $f$ is linear in $\mathbf{q}$, no second-order derivative in this component exits. Also, the quadratic variance of $b(\varrho)$ is zero since the renormalized continuity equation is deterministic.

Now, notice that the Bogovskil operator commutes with the time derivative (but not with the spatial derivative) and since the continuity equation is satisfied in the renormalized sense, we have that

$$
\mathrm{d}\left[\mathbb{B}\left(b\left(\varrho_{L}\right)\right)\right]=\mathbb{B}\left[\mathrm{d}\left(b\left(\varrho_{L}\right)\right)\right]=-\mathbb{B}\left[\operatorname{div}\left(b\left(\varrho_{L}\right) \mathrm{u}_{L}\right)-\left(b^{\prime}\left(\varrho_{L} \varrho_{\varrho_{L}}-b\left(\varrho_{L}\right)\right) \operatorname{div} \mathrm{u}_{L}\right] \mathrm{d} t .\right.
$$

As such for $\mathrm{b}_{L}:=b\left(\varrho_{L}\right)$, the following holds in expectation:

$$
\begin{aligned}
& \int_{0}^{t} f_{b_{L}}\left(b_{L}, \mathbf{q}_{L}\right) \mathrm{d} b_{L}=\iint \mathbf{q}_{L} \cdot \partial_{b_{L}}\left(\mathbb{B}\left(b_{L}\right)\right) \mathrm{d} b_{L} \mathrm{~d} x=\iint \mathbf{q}_{L} \cdot \mathrm{d}\left[\mathbb{B}\left(b_{L}\right)\right] \mathrm{d} x \\
& =-\iint \mathbf{q}_{L} \cdot \mathbb{B}\left[\operatorname{div}\left(b_{L} \mathbf{u}_{L}\right)\right] \mathrm{d} x \mathrm{~d} s-\iint \mathbf{q}_{L} \cdot \mathbb{B}\left[\left(\varrho_{L} b_{L}^{\prime}-b_{L}\right) \operatorname{div} \mathbf{u}_{L}\right] \mathrm{d} x \mathrm{~d} s \\
& \int_{0}^{t} f_{\mathbf{q}_{L}}\left(b_{L}, \mathbf{q}_{L}\right) \mathrm{d} \mathbf{q}_{L} \\
& =\iint \mathbb{B}\left(b_{L}\right) \mathrm{d} \mathbf{q}_{L} \mathrm{~d} x \\
& =\iint \mathbb{B}\left(b_{L}\right)\left[-\operatorname{div}\left(\varrho_{L} \mathbf{u}_{L} \otimes \mathbf{u}_{L}\right)+v \Delta \mathbf{u}_{L}+(\lambda+v) \nabla \operatorname{div} \mathbf{u}_{L}-a \nabla \varrho_{L}^{\gamma}\right] \mathrm{d} x \mathrm{~d} s \\
& \quad+\iint \mathbb{B}\left(b_{L}\right) \Phi\left(\varrho_{L}, \varrho_{L} \mathbf{u}_{L}\right) \mathrm{d} W \mathrm{~d} x \\
& =\iint\left[\left(\varrho_{L} \mathbf{u}_{L} \otimes \mathbf{u}_{L}\right) \nabla \mathbb{B}\left(b_{L}\right) \mathrm{d} x \mathrm{~d} s-v \nabla \mathbf{u}_{L}: \nabla \mathbb{B}\left(b_{L}\right)-(\lambda+v) b_{L} \mathrm{div} \mathbf{u}_{L}\right] \mathrm{d} x \mathrm{~d} s \\
& \quad+\iint a \varrho_{L}^{\gamma} b_{L} \mathrm{~d} x \mathrm{~d} s+\iint \mathbb{B}\left(b_{L}\right) \Phi\left(\varrho_{L}, \varrho_{L} \mathbf{u}_{L}\right) \mathrm{d} W \mathrm{~d} x \\
& \quad \int_{0}^{t} f_{b_{L} b_{L}}\left(b_{L}, \mathbf{q}_{L}\right) \mathrm{d}\left\langle b_{L}\right\rangle \\
& =\int_{0}^{t} f_{\mathbf{q}_{L} \mathbf{q}_{L}}\left(b_{L}, \mathbf{q}_{L}\right) \mathrm{d}\left\langle\mathbf{q}_{L}\right\rangle=0 \text { since } \mathrm{d}\left\langle b_{L}\right\rangle=f_{\mathbf{q}_{L} \mathbf{q}_{L}}=0
\end{aligned}
$$

where we have integrated by parts and used the fact that $\mathbb{B}(f)$ solves the equation $\operatorname{div} \mathbf{v}=f$. It therefore follows that

$$
\begin{gathered}
\mathbb{E} \int_{B_{r, L}^{3}} \mathbf{q}_{L} \cdot \mathbb{B}\left(b_{L}\right) \mathrm{d} x=\mathbb{E} \int_{B_{r, L}^{3}} \mathbf{q}_{L}(0) \cdot \mathbb{B}\left[b_{L}(0)\right] \mathrm{d} x \\
-\mathbb{E} \int_{0}^{t} \int_{B_{r, L}^{3}} \mathbf{q}_{L} \cdot \mathbb{B}\left[\operatorname{div}\left(b_{L} \mathbf{u}_{L}\right)\right] \mathrm{d} x \mathrm{~d} s
\end{gathered}
$$




$$
\begin{aligned}
& -\mathbb{E} \int_{0}^{t} \int_{B_{r, L}^{3}} \mathbf{q}_{L} \cdot \mathbb{B}\left[\varrho_{L} b_{L}^{\prime} \operatorname{div} \mathbf{u}_{L}\right] \mathrm{d} x \mathrm{~d} s+\mathbb{E} \int_{0}^{t} \int_{B_{r, L}^{3}} \mathbf{q}_{L} \cdot \mathbb{B}\left[b_{L} \operatorname{div} \mathbf{u}_{L}\right] \mathrm{d} x \mathrm{~d} s \\
& +\mathbb{E} \int_{0}^{t} \int_{B_{r, L}^{3}}\left(\varrho_{L} \mathbf{u}_{L} \otimes \mathbf{u}_{L}\right) \nabla \mathbb{B}\left(b_{L}\right) \mathrm{d} x \mathrm{~d} s-\mathbb{E} \int_{0}^{t} \int_{B_{r, L}^{3}} \nu \nabla \mathbf{u}_{L}: \nabla \mathbb{B}\left(b_{L}\right) \mathrm{d} x \mathrm{~d} s \\
& -\mathbb{E} \int_{0}^{t} \int_{B_{r, L}^{3}}(\lambda+v) b_{L} \operatorname{div} \mathbf{u}_{L} \mathrm{~d} x \mathrm{~d} s+\mathbb{E} \int_{0}^{t} \int_{B_{r, L}^{3}} a \varrho_{L}^{\gamma} b_{L} \mathrm{~d} x \mathrm{~d} s \\
& +\mathbb{E} \int_{0}^{t} \int_{B_{r, L}^{3}} \mathbb{B}\left(b_{L}\right) \Phi\left(\varrho_{L}, \varrho_{L} \mathbf{u}_{L}\right) \mathrm{d} W \mathrm{~d} x=: \mathbb{E} \sum_{i=1}^{9} J_{i} .
\end{aligned}
$$

To improve the regularity of $\varrho$, we aim at estimating $J_{8}$ in terms of the rest.

To do this, we first set the left-hand side of (27) to $\mathbb{E} J_{0}$. Then using (8), (19), (22), (23) and heavy reliance on Hölder inequalities, we can show just as in [5, Propositions 5.1, 6.1] for $\delta=0$ and noting that $\Delta^{-1} \nabla$ and $\mathbb{B}$ enjoys the same continuity properties;

$$
\mathbb{E} J_{i} \leq c, \quad \text { for all } i \in\{0,1,2,3,4,5,6,7,9\}
$$

for some constants $c=c_{\Theta, \gamma}$ which are in particular, independent of $L$.

Remark 5 In estimating $J_{2}$, we use instead the Bogovskil operator in negative spaces which can be found in [18, Proposition 2.1], [2,10].

Also, note the comment just after [18, Remark 2.2] about carrying over the properties of the Bogovskiı̌ operator from a star shaped domain onto more common domains treated in the analysis of PDE's.

The result follows by making $\mathbb{E} J_{8}$ the subject and estimating it from above with the estimates given by the rest.

\subsection{Compactness}

We now show that not only are our earlier estimates bounded uniformly in the torus $\mathbb{T}_{L}^{3}$ but due to the fact that each constants obtained are uniform in $L$, they are indeed bounded locally in the whole space $\mathbb{R}^{3}$. We then proceed to show the usual compactness arguments.

Lemma 2 For any $L \geq 1$, we have that

$$
\begin{array}{cc}
\mathbf{u}_{L} \in L^{p}\left(\Omega ; L^{2}\left(0, T ; W_{\mathrm{loc}}^{1,2}\left(\mathbb{R}^{3}\right)\right)\right), & \sqrt{\varrho_{L}} \mathbf{u}_{L} \in L^{p}\left(\Omega ; L^{\infty}\left(0, T ; L_{\mathrm{loc}}^{2}\left(\mathbb{R}^{3}\right)\right)\right), \\
\varrho_{L} \in L^{p}\left(\Omega ; L^{\infty}\left(0, T ; L_{\mathrm{loc}}^{\gamma}\left(\mathbb{R}^{3}\right)\right)\right), & \varrho_{L} \mathbf{u}_{L} \in L^{p}\left(\Omega ; L^{\infty}\left(0, T ; L_{\mathrm{loc}}^{\frac{2 \gamma}{\gamma+1}}\left(\mathbb{R}^{3}\right)\right)\right), \\
\varrho_{L} \mathbf{u}_{L} \otimes \mathbf{u}_{L} \in L^{p}\left(\Omega ; L^{2}\left(0, T ; L_{\mathrm{loc}}^{\frac{6 \gamma}{4 \gamma+3}}\left(\mathbb{R}^{3}\right)\right)\right), & \varrho_{L} \in L^{p}\left(\Omega ; L^{\gamma+\Theta}\left(0, T ; L_{\mathrm{loc}}^{\gamma+\Theta}\left(\mathbb{R}^{3}\right)\right)\right) .
\end{array}
$$

uniformly in L.

Proof We will only show the first uniform estimate as the rest can be done in exactly the same manner in conjunction with (19), (23) and Lemma 1.

Let $L, r \in \mathbb{N}$ and let $B_{r} \subset \mathbb{R}^{3}$ be the ball of radius $r$ centered at the origin. If $B_{r} \subset \mathbb{T}_{L}^{3}$, then we notice that we can directly deduce from $(19)_{2}$ that

$$
\mathbf{u}_{L} \in L^{p}\left(\Omega ; L^{2}\left(0, T ; W^{1,2}\left(B_{r}\right)\right)\right)
$$


uniformly in $L$. Otherwise, we can use the same argument as in the justification of (20) above to get from $(19)_{2}$,

$$
\left\|\mathbf{u}_{L}\right\|_{L^{p}\left(\Omega ; L^{2}\left(0, T ; W^{1,2}\left(B_{r}\right)\right)\right)} \leq c(p, r), \quad \forall r \in \mathbb{N}
$$

uniformly in $L$. That is, for any $r \in \mathbb{N}$ and any $B_{r} \subset \mathbb{R}^{3},(29)$ holds. By combining (28) and (29), we can deduce that

$$
\mathbf{u}_{L} \in L^{p}\left(\Omega ; L^{2}\left(0, T ; W_{\text {loc }}^{1,2}\left(\mathbb{R}^{3}\right)\right)\right)
$$

uniformly in $L$.

For the compactness result, let define the following path space $\chi=\chi_{\mathbf{u}} \times \chi_{\varrho} \times \chi_{\varrho \mathbf{u}} \times \chi_{W}$ where

$$
\begin{aligned}
\chi_{\mathbf{u}} & =\left(L^{2}\left(0, T ; W_{\mathrm{loc}}^{1,2}\left(\mathbb{R}^{3}\right)\right), \omega\right), \\
\chi_{\varrho} & =C_{\omega}\left([0, T] ; L_{\mathrm{loc}}^{\gamma}\left(\mathbb{R}^{3}\right)\right) \cap\left(L^{\gamma+\theta}\left(0, T ; L_{\mathrm{loc}}^{\gamma+\theta}\left(\mathbb{R}^{3}\right)\right), \omega\right), \\
\chi_{\varrho \mathbf{u}} & =C_{\omega}\left([0, T] ; L_{\mathrm{loc}}^{\frac{2 \gamma}{\gamma+1}}\left(\mathbb{R}^{3}\right)\right), \\
\chi_{W} & =C\left([0, T] ; \mathfrak{U}_{0}\right)
\end{aligned}
$$

and let

1. $\mu_{\mathbf{u}_{L}}$ be the law of $\mathbf{u}_{L}$ on $\chi_{\mathbf{u}}$,

2. $\mu_{\varrho_{L}}$ be the law of $\varrho_{L}$ on the space $\chi_{\varrho}$,

3. $\mu_{\varrho_{L} \mathbf{u}_{L}}$ be the law of $\varrho_{L} \mathbf{u}_{L}$ on the space $\chi_{\varrho \mathbf{u}}$,

4. $\mu_{W}$ be the law of $W$ on the space $\chi_{W}$,

5. $\mu^{L}$ be the joint law of $\mathbf{u}_{L}, \varrho_{L}, \varrho_{L} \mathbf{u}_{L}$ and $W$ on the space $\chi$.

Proposition 1 For an arbitrary constant $c$, which is uniform in $r \in \mathbb{N}, L \geq 1$ and $R>0$, let us define the set

$$
A_{R}:=\left\{\mathbf{u}_{L} \in L^{2}\left(0, T ; W_{\mathrm{loc}}^{1,2}\left(\mathbb{R}^{3}\right)\right):\left\|\mathbf{u}_{L}\right\|_{L^{2}\left(0, T ; W^{1,2}\left(B_{r}\right)\right)} \leq c(r) R, \quad \forall r \in \mathbb{N}\right\} .
$$

Then $A_{R}$ is compact in $\chi_{\mathbf{u}}$.

Proof To see this, fix $R>0$ and consider the sequence $\left\{\mathbf{u}_{n}\right\}_{n \in \mathbb{N}} \subset A_{R}$ so that

$$
\left\|\mathbf{u}_{n}\right\|_{L^{2}\left(0, T ; W^{1,2}\left(B_{r}\right)\right)} \leq c(r) R, \quad \forall n \in \mathbb{N} \text { and } \forall r \in \mathbb{N}
$$

Then by the use of a diagonal argument, we can construct the sequence $\left\{\mathbf{u}_{n}^{n}\right\}_{n \in \mathbb{N}} \subset\left\{\mathbf{u}_{n}\right\}_{n \in \mathbb{N}}$ that is a common subsequence of all the sequences $\left\{\mathbf{u}_{n}^{m}\right\}_{n \in \mathbb{N}}$ for all $m \in\{0\} \cup \mathbb{N}$ where $\mathbf{u}_{n}^{0}:=\mathbf{u}_{n}$. And by uniqueness of limits, we can therefore conclude that

$$
\mathbf{u}_{n}^{n} \rightarrow \mathbf{u} \text { in } L^{2}\left(0, T ; W^{1,2}\left(B_{r}\right)\right) \text { for every } r \in \mathbb{N} .
$$

This finishes the proof.

Proposition 2 The family of measures $\left\{\mu^{L} ; L \geq 1\right\}$ is tight on $\chi$.

Proof We first show that $\left\{\mu_{\mathbf{u}_{L}} ; L \geq 1\right\}$ is tight on $\chi_{\mathbf{u}}$. To do this, we let $R>0$, then by Proposition 1, there exists a compact subset $A_{R} \subset \chi_{\mathbf{u}}$. Now since

$$
\left(A_{R}\right)^{C}:=\left\{\mathbf{u}_{L} \in L^{2}\left(0, T ; W_{\mathrm{loc}}^{1,2}\left(\mathbb{R}^{3}\right)\right):\left\|\mathbf{u}_{L}\right\|_{L^{2}\left(0, T ; W^{1,2}\left(B_{r}\right)\right)}>c(r) R, \text { for some } r \in \mathbb{N}\right\},
$$


for any measure $\mu_{\mathbf{u}_{L}} \in\left\{\mu_{\mathbf{u}_{L}} ; L \geq 1\right\}$, there exists a $r \in \mathbb{N}$ such that:

$$
\begin{aligned}
\mu_{\mathbf{u}_{L}}\left(\left(A_{R}\right)^{C}\right) & =\mathbb{P}\left(\left\|\mathbf{u}_{L}\right\|_{L^{2}\left(0, T ; W^{1,2}\left(B_{r}\right)\right)}>c(r) R\right) \\
& <\frac{1}{c(r) R} \mathbb{E}\left(\left\|\mathbf{u}_{L}\right\|_{L^{2}\left(0, T ; W^{1,2}\left(B_{r}\right)\right)}\right) \leq \frac{1}{R} \rightarrow 0 .
\end{aligned}
$$

as $R \rightarrow \infty$, where we have used (29) in the last inequality. This implies that $\left\{\mu_{\mathbf{u}_{L}} ; L \geq 1\right\}$ is tight on $\chi_{\mathbf{u}}$.

By using a similar argument adapted to suit the compactness arguments in [5, Sect. 6] we can show that $\left\{\mu_{\varrho_{L}} ; L \geq 1\right\}$ and $\left\{\mu_{\varrho_{L} \mathbf{u}_{L}} ; L \geq 1\right\}$ are also tight on $\chi_{\varrho}$ and $\chi_{\varrho u}$, respectively. Furthermore, $\mu_{W}$ is tight since its a Radon measure on the Polish space $\chi_{W}$. This finishes the proof.

From Proposition 2, we cannot immediately use Skorokhod representation theorem to deduce that $\left\{\mu^{L} ; L \geq 1\right\}$ is relatively compact (i.e., Prokhorov theorem), since the path space $\chi$ is not metrizable. However, we may use instead the Jakubowski-Skorokhod representation theorem [20] that gives a similar result but for more general spaces including quasi-Polish spaces, the space in which these locally in space Sobolev functions live. Applying this yields the following result:

Proposition 3 There exists a subsequence $\mu^{n}:=\mu^{L_{n}}$ for $n \in \mathbb{N}$, a probability space $(\tilde{\Omega}, \tilde{\mathscr{F}}, \tilde{\mathbb{P}})$ with $\chi$-valued random variables $\left(\tilde{\mathbf{u}}_{n}, \tilde{\varrho}_{n}, \tilde{\mathbf{q}}_{n}, \tilde{W}_{n}\right)$, and their corresponding 'limit' variables $(\tilde{\mathbf{u}}, \tilde{\varrho}, \tilde{\mathbf{q}}, \tilde{W})$ such that

- the law of $\left(\tilde{\mathbf{u}}_{n}, \tilde{\varrho}_{n}, \tilde{\mathbf{q}}_{n}, \tilde{W}_{n}\right)$ is given by $\mu^{n}=\operatorname{Law}\left(\mathbf{u}_{L_{n}}, \varrho_{L_{n}}, \varrho_{L_{n}} \mathbf{u}_{L_{n}}, W\right), n \in \mathbb{N}$,

- the law of $(\tilde{\mathbf{u}}, \tilde{\varrho}, \tilde{\mathbf{q}}, \tilde{W})$, denoted by $\mu=\operatorname{Law}(\mathbf{u}, \varrho, \varrho \mathbf{u}, W)$ is a Randon measure,

- $\left(\tilde{\mathbf{u}}_{n}, \tilde{\varrho}_{n}, \tilde{\mathbf{q}}_{n}, \tilde{W}_{n}\right)$ converges $\tilde{\mathbb{P}}-$ a.s to $(\tilde{\mathbf{u}}, \tilde{\varrho}, \tilde{\mathbf{q}}, \tilde{W})$ in the topology of $\chi$.

To extend this new probability space $(\tilde{\Omega}, \tilde{\mathscr{F}}, \tilde{\mathbb{P}})$ into a stochastic basis, we endow it with a filtration. To do this, let us first define a restriction operator $\mathbf{r}_{t}$ define by

$$
\mathbf{r}_{t}:\left.X \rightarrow X\right|_{[0, t]},\left.\quad f \mapsto f\right|_{[0, t]},
$$

for $t \in[0, T]$ and $X \in\left\{\chi_{\varrho}, \chi_{\mathbf{u}}, \chi_{W}\right\}$. We observe that $\mathbf{r}_{t}$ is a continuous map. We can therefore construct $\tilde{\mathbb{P}}$-augmented canonical filtrations for $\left(\tilde{\varrho}_{n}, \tilde{\mathbf{u}}_{n}, \tilde{W}_{n}\right)$ and $(\tilde{\varrho}, \tilde{\mathbf{u}}, \tilde{W})$, respectively, by setting

$$
\begin{aligned}
& \tilde{\mathscr{F}}_{t}^{n}=\sigma\left(\sigma\left(\mathbf{r}_{t} \tilde{\varrho}_{n}, \mathbf{r}_{t} \tilde{\mathbf{u}}_{n}, \mathbf{r}_{t} \tilde{W}_{n}\right) \cup\{N \in \tilde{\mathscr{F}} ; \tilde{\mathbb{P}}(N)=0\}\right), \quad t \in[0, T], \\
& \tilde{\mathscr{F}}_{t}=\sigma\left(\sigma\left(\mathbf{r}_{t} \tilde{\varrho}, \mathbf{r}_{t} \tilde{\mathbf{u}}, \mathbf{r}_{t} \tilde{W}\right) \cup\{N \in \tilde{\mathscr{F}} ; \tilde{\mathbb{P}}(N)=0\}\right), \quad t \in[0, T] .
\end{aligned}
$$

The following result thus follows:

Lemma 3 For any $n>0,\left[\left(\tilde{\Omega}, \tilde{\mathscr{F}},\left(\tilde{\mathscr{F}}_{t}^{n}\right)_{t \geq 0}, \tilde{\mathbb{P}}\right), \tilde{\varrho}_{n}, \tilde{\mathbf{u}}_{n}, \tilde{W}_{n}\right]$ is a weak martingale solution of (4) with initial law $\Lambda$. Furthermore, there exists $b>\frac{3}{2}$ and $a W^{-b, 2}\left(\mathbb{R}^{3}\right)$-valued continuous square integrable $\left(\tilde{\mathscr{F}}_{t}\right)$-martingale $\tilde{M}$ and $\tilde{p} \in L^{\frac{\gamma+\Theta}{\gamma}}(\tilde{\Omega} \times Q)$, where $Q=$ $(0, T) \times \mathbb{R}^{3}$, such that $\left[\left(\tilde{\Omega}, \tilde{\mathscr{F}},\left(\tilde{\mathscr{F}}_{t}\right)_{t \geq 0}, \tilde{\mathbb{P}}\right), \tilde{\varrho}, \tilde{\mathbf{u}}, \tilde{p}, \tilde{M}\right]$ is a weak martingale solution of

$$
\begin{gathered}
\mathrm{d} \tilde{\varrho}+\operatorname{div}(\tilde{\varrho} \tilde{\mathbf{u}}) \mathrm{d} t=0 \\
\mathrm{~d}(\tilde{\varrho} \tilde{\mathbf{u}})+[\operatorname{div}(\tilde{\varrho} \tilde{\mathbf{u}} \otimes \tilde{\mathbf{u}})-v \Delta \tilde{\mathbf{u}}-(\lambda+v) \nabla \operatorname{div} \tilde{\mathbf{u}}+\nabla \tilde{p}] \mathrm{d} t=\mathrm{d} \tilde{M}, \quad \text { in } \tilde{\Omega} \times Q
\end{gathered}
$$

with initial law $\Lambda$. Furthermore, (32) 1 is satisfied in the renormalized sense. 
Proof This follows in exactly the same manner as in [5, prop 5.6].

Corollary 1 The following $\tilde{\mathbb{P}}-$ a.s. convergence holds:

$$
\begin{array}{rll}
\tilde{\mathbf{u}}_{n} \rightarrow \tilde{\mathbf{u}} & \text { in } & L^{2}\left(0, T ; W_{\mathrm{loc}}^{1,2}\left(\mathbb{R}^{3}\right)\right), \\
\tilde{\varrho}_{n} \rightarrow \tilde{\varrho} \quad \text { in } \quad & C_{\omega}\left([0, T] ; L_{\mathrm{loc}}^{\gamma}\left(\mathbb{R}^{3}\right)\right), \\
\tilde{\varrho}_{n} \rightarrow \tilde{\varrho} & \text { in } \quad L^{\gamma+\Theta}\left(0, T ; L_{\mathrm{loc}}^{\gamma+\Theta}\left(\mathbb{R}^{3}\right)\right), \\
\tilde{\varrho}_{n} \tilde{\mathbf{u}}_{n} \rightarrow \tilde{\varrho} \tilde{\mathbf{u}} & \text { in } \quad & C_{\omega}\left([0, T] ; L_{\mathrm{loc}}^{\frac{2 \gamma}{\gamma+1}}\left(\mathbb{R}^{3}\right)\right) \cap L^{2}\left(0, T ; W_{\mathrm{loc}}^{-1,2}\left(\mathbb{R}^{3}\right)\right) \\
\tilde{\varrho}_{n} \tilde{\mathbf{u}}_{n} \otimes \tilde{\mathbf{u}}_{n} \rightarrow \tilde{\varrho} \tilde{\mathbf{u}} \otimes \tilde{\mathbf{u}} & \text { in } \quad L^{1}\left(0, T ; L_{\mathrm{loc}}^{1}\left(\mathbb{R}^{3}\right)\right), \\
\tilde{W}_{n} \rightarrow \tilde{W} \quad \text { in } & C\left([0, T] ; \mathfrak{U}_{0}\right),
\end{array}
$$

Proof The first three and the last is exactly consisted in Proposition 3. For $(33)_{4,5}$, see [5, Lemma 5.5, Proposition 6.3].

Proposition 4 The limit processes $\tilde{\mathbf{u}}$ in (33) is globally defined in space, i.e., $\tilde{\mathbf{u}} \in$ $L^{2}\left(0, T ; W^{1,2}\left(\mathbb{R}^{3}\right)\right)$

Proof Let $B_{r} \subset \mathbb{R}^{3}$ be an arbitrary ball of radius $r>0$. Then from (33) $)_{1}$, we have that for $\tilde{\mathbb{P}}$ - a.s.,

$$
\tilde{\mathbf{u}}_{n} \rightarrow \tilde{\mathbf{u}} \text { in } L^{2}\left(0, T ; W^{1,2}\left(B_{r}\right)\right), \text { for } r>0 .
$$

However, lower semicontinuity of norms means that for any such $r>0$,

$$
\left\|\chi_{B_{r}} \nabla \tilde{\mathbf{u}}\right\|_{L^{2}\left(0, T ; L^{2}\left(\mathbb{R}^{3}\right)\right)}=\|\nabla \tilde{\mathbf{u}}\|_{L^{2}\left(0, T ; L^{2}\left(B_{r}\right)\right)} \leq \liminf _{n \rightarrow \infty}\left\|\nabla \tilde{\mathbf{u}}_{n}\right\|_{L^{2}\left(0, T ; L^{2}\left(B_{r}\right)\right)}
$$

$\tilde{\mathbb{P}}$-a.s. Passing to the limit $r \rightarrow \infty$ on either side of this inequality finishes the proof since by the Gagliardo-Nirenberg-Sobolev inequality, (8) then follows for $q=2$.

\subsection{The effective viscous flux}

This section combines ideas from [5,15] and [28, Chapter 7].

Let $\Delta^{-1}$ be the inverse Laplacian on $\mathbb{R}^{3}$ and let the global-in-space operators $\mathcal{A}_{i}=$ $\Delta^{-1}\left[\partial_{x_{i}} u\right], \quad i=1,2,3$ be as defined in [28, Sect. 4.4.1] or [15, Sect. 3.4].

Then by using the convention $\partial_{i}:=\partial_{x_{i}}$ and for some cutoff functions $\phi(x), \phi(x) \in$ $C_{c}^{\infty}\left(\mathbb{R}^{3}\right)$, we may do a similar computation as in (27). That is, we apply Itô's formula to the function $f(g, \tilde{\mathbf{q}})=\int_{\mathbb{R}^{3}} \tilde{\mathbf{q}} \cdot \phi(x) \mathcal{A}_{i}[\underline{\phi}(x) g] \mathrm{d} x$ where $\tilde{\mathbf{q}}=\tilde{\varrho} \tilde{\mathbf{u}}$ and where $g=T_{k}(\tilde{\varrho})$ and $T_{k}:[0, \infty) \rightarrow[0, \infty)$ is given by

$$
T_{k}(t)= \begin{cases}t & \text { if } 0 \leq t<k \\ k & \text { if } k \leq t<\infty\end{cases}
$$

Or equivalently, by testing the momentum equation satisfied by the sequence of weak martingale solution in Lemma 3 by $\varphi_{i}(x)=\phi(x) \mathcal{A}_{i}\left[\phi(x) T_{k}(\tilde{\varrho})\right]$. We obtain the following (by assuming that $L$ is large enough such that $\operatorname{spt} \phi \subset \overline{\mathbb{T}}_{L}^{3}$ )

$$
\begin{aligned}
\tilde{\mathbb{E}} \int_{\mathbb{R}^{3}} \phi \tilde{\varrho}_{n} \tilde{u}_{n}^{i} \mathcal{A}_{i}\left[\phi T_{k}\left(\tilde{\varrho}_{n}\right)\right] \mathrm{d} x= & \tilde{\mathbb{E}} \int_{\mathbb{R}^{3}} \phi \tilde{\varrho}_{n} \tilde{u}_{n}^{i}(0) \mathcal{A}_{i}\left[\phi T_{k}\left(\tilde{\varrho}_{n}(0)\right)\right] \mathrm{d} x \\
& -\tilde{\mathbb{E}} \int_{0}^{t} \int_{\mathbb{R}^{3}} \phi \tilde{\varrho}_{n} \tilde{u}_{n}^{i} \mathcal{A}_{i}\left[\underline{\phi} \partial_{j}\left(T_{k}\left(\tilde{\varrho}_{n}\right) \tilde{u}_{n}^{j}\right)\right] \mathrm{d} x \mathrm{~d} s
\end{aligned}
$$




$$
\begin{aligned}
& -\tilde{\mathbb{E}} \int_{0}^{t} \int_{\mathbb{R}^{3}} \phi \tilde{\varrho}_{n} \tilde{u}_{n}^{i} \mathcal{A}_{i}\left[\underline { \phi } \left(T_{k}^{\prime}\left(\tilde{\varrho}_{n}\right) \tilde{\varrho}_{n}\right.\right. \\
& \left.\left.-T_{k}\left(\tilde{\varrho}_{n}\right)\right) \operatorname{div} \tilde{\mathbf{u}}_{n}\right] \mathrm{d} x \mathrm{~d} s \\
& +\tilde{\mathbb{E}} \int_{0}^{t} \int_{\mathbb{R}^{3}} \tilde{\varrho}_{n} \tilde{u}_{n}^{i} \tilde{u}_{n}^{j} \partial_{j}\left(\phi \mathcal{A}_{i}\left[\phi T_{k}\left(\tilde{\varrho}_{n}\right)\right]\right) \mathrm{d} x \mathrm{~d} s \\
& +v \tilde{\mathbb{E}} \int_{0}^{t} \int_{\mathbb{R}^{3}} \phi \mathcal{A}_{i}\left[\underline{\phi} T_{k}\left(\tilde{\varrho}_{n}\right)\right] \Delta \tilde{u}_{n}^{i} \mathrm{~d} x \mathrm{~d} s \\
& +\tilde{\mathbb{E}} \int_{0}^{t} \int_{\mathbb{R}^{3}}\left[a \tilde{\varrho}_{n}^{\gamma}-(\lambda+v) \operatorname{div} \tilde{\mathbf{u}}_{n}\right] \partial_{i}\left(\phi \mathcal{A}_{i}\left[\phi \underline{T}_{k}\left(\tilde{\varrho}_{n}\right)\right]\right) \mathrm{d} x \mathrm{~d} s \\
& =: \tilde{\mathbb{E}} \sum_{k=1}^{6} J_{k}, \quad i=1,2,3 \text {. }
\end{aligned}
$$

where $T_{k}$, as defined above, and replaces $b$ in the definition of the renormalized equation defined in (12).

Remark 6 Notice that since our convergence results for the approximate quantities in (33) are only obtained locally in space, to apply this globally defined operators $\mathcal{A}$, it is essentially to pre-multiply our functions by some $\phi \in C_{c}^{\infty}(\mathbb{R})$.

Also, we observe that since our noise term is a martingale, it vanishes when we take its expectation, since martingales are constant on average.

Now notice that by integration by parts and the use of the properties of the operators $\mathcal{A}_{i}$ and $\mathcal{R}_{i j}=\partial_{i} \mathcal{A}_{j}$, we may rewrite $J_{2}, J_{4}, J_{5}$ and $J_{6}$ so that (34) becomes:

$$
\begin{aligned}
& \tilde{\mathbb{E}} \int_{0}^{t} \int_{\mathbb{R}^{3}}\left[a \tilde{\varrho}_{n}^{\gamma}-(\lambda+2 v) \operatorname{div} \tilde{\mathbf{u}}_{n}\right] \phi \underline{\phi} T_{k}\left(\tilde{\varrho}_{n}\right) \mathrm{d} x \mathrm{~d} s \\
&=\tilde{\mathbb{E}} \int_{\mathbb{R}^{3}} \phi \tilde{\varrho}_{n} \tilde{u}_{n}^{i} \mathcal{A}_{i}\left[\underline{\phi} T_{k}\left(\tilde{\varrho}_{n}\right)\right] \mathrm{d} x \\
& \quad-\tilde{\mathbb{E}} \int_{\mathbb{R}^{3}} \phi \tilde{\varrho}_{n} \tilde{u}_{n}^{i}(0) \mathcal{A}_{i}\left[\underline{\phi} T_{k}\left(\tilde{\varrho}_{n}(0)\right)\right] \mathrm{d} x+v \tilde{\mathbb{E}}_{0}^{t} \int_{\mathbb{R}^{3}} \underline{\phi} \tilde{u}_{n}^{i} T_{k}\left(\tilde{\varrho}_{n}\right) \partial_{i} \phi \mathrm{d} x \mathrm{~d} s \\
&-\tilde{\mathbb{E}} \int_{0}^{t} \int_{\mathbb{R}^{3}}\left[a \tilde{\varrho}_{n}^{\gamma}-(\lambda+v) \operatorname{div} \tilde{\mathbf{u}}_{n}\right] \mathcal{A}_{i}\left[\underline{\phi} T_{k}\left(\tilde{\varrho}_{n}\right)\right] \partial_{i} \phi \mathrm{d} x \mathrm{~d} s \\
&+\tilde{\mathbb{E}} \int_{0}^{t} \int_{\mathbb{R}^{3}} \phi \tilde{\varrho}_{n} \tilde{u}_{n}^{i} \mathcal{A}_{i}\left[\underline{\phi}\left(T_{k}^{\prime}\left(\tilde{\varrho}_{n}\right) \tilde{\varrho}_{n}-T_{k}\left(\tilde{\varrho}_{n}\right)\right) \operatorname{div} \tilde{\mathbf{u}}_{n}\right] \mathrm{d} x \mathrm{~d} s \\
&+\tilde{\mathbb{E}} \int_{0}^{t} \int_{\mathbb{R}^{3}} \tilde{u}_{n}^{i}\left(\mathcal{R}_{i j}\left[\phi \tilde{\varrho}_{n} \tilde{u}_{n}^{j}\right] \underline{\phi} T_{k}\left(\tilde{\varrho}_{n}\right)-\phi \tilde{\varrho}_{n} \tilde{u}_{n}^{j} \mathcal{R}_{i j}\left[\underline{\phi} T_{k}\left(\tilde{\varrho}_{n}\right)\right]\right) \mathrm{d} x \mathrm{~d} s \\
&+\tilde{\mathbb{E}} \int_{0}^{t} \int_{\mathbb{R}^{3}} \tilde{u}_{n}^{j}\left(\mathcal{A}_{i}\left[\phi \tilde{\varrho}_{n} \tilde{u}_{n}^{i}\right] T_{k}\left(\tilde{\varrho}_{n}\right) \partial_{j} \phi-\tilde{\varrho}_{n} \tilde{u}_{n}^{i} \mathcal{A}_{i}\left[\phi T_{k}\left(\tilde{\varrho}_{n}\right)\right] \partial_{j} \phi\right) \mathrm{d} x \mathrm{~d} s \\
&=: \tilde{\mathbb{E}} \sum_{k=1}^{7} I_{k}, \quad i=1,2,3 .
\end{aligned}
$$

Remark 7 If we set the left-hand side of (35) to $\tilde{\mathbb{E}} I_{0}$, then we point the reader to the difference in the viscosity constant in $I_{0}$ and $I_{4}$. 
Similarly for the limit processes, we obtain

$$
\begin{aligned}
& \tilde{\mathbb{E}} \int_{0}^{t} \int_{\mathbb{R}^{3}}[a \tilde{p}-(\lambda+2 v) \operatorname{div} \tilde{\mathbf{u}}] \phi \underline{\phi} \overline{T_{k}(\tilde{\varrho})} \mathrm{d} x \mathrm{~d} s=\tilde{\mathbb{E}} \int_{\mathbb{R}^{3}} \phi \tilde{\varrho} \tilde{u}^{i} \mathcal{A}_{i}\left[\underline{\phi} \overline{T_{k}(\tilde{\varrho})}\right] \mathrm{d} x \\
& -\tilde{\mathbb{E}} \int_{\mathbb{R}^{3}} \phi \tilde{\varrho} \tilde{u}^{i}(0) \mathcal{A}_{i}\left[\underline{\phi} \overline{T_{k}(\tilde{\varrho}(0))}\right] \mathrm{d} x+v \tilde{\mathbb{E}} \int_{0}^{t} \int_{\mathbb{R}^{3}} \phi \tilde{u}^{i} \overline{T_{k}(\tilde{\varrho})} \partial_{i} \phi \mathrm{d} x \mathrm{~d} s \\
& -\tilde{\mathbb{E}} \int_{0}^{t} \int_{\mathbb{R}^{3}}[a \overline{\tilde{p}}-(\lambda+v) \operatorname{div} \tilde{\mathbf{u}}] \mathcal{A}_{i}\left[\underline{\phi} \overline{T_{k}(\tilde{\varrho})}\right] \partial_{i} \phi \mathrm{d} x \mathrm{~d} s \\
& +\tilde{\mathbb{E}} \int_{0}^{t} \int_{\mathbb{R}^{3}} \phi \tilde{\varrho} \tilde{u}^{i} \mathcal{A}_{i}\left[\underline{\phi} \overline{\left(T_{k}^{\prime}(\tilde{\varrho}) \tilde{\varrho}-T_{k}(\tilde{\varrho})\right) \operatorname{div} \tilde{\mathbf{u}}}\right] \mathrm{d} x \mathrm{~d} s \\
& +\tilde{\mathbb{E}} \int_{0}^{t} \int_{\mathbb{R}^{3}} \tilde{u}^{i}\left(\mathcal{R}_{i j}\left[\phi \tilde{\varrho} \tilde{u}^{j}\right] \underline{\phi} \overline{T_{k}(\tilde{\varrho})}-\phi \tilde{\varrho} \tilde{u}^{j} \mathcal{R}_{i j}\left[\underline{\phi} \overline{T_{k}(\tilde{\varrho})}\right]\right) \mathrm{d} x \mathrm{~d} s \\
& +\tilde{\mathbb{E}} \int_{0}^{t} \int_{\mathbb{R}^{3}} \tilde{u}^{j}\left(\mathcal{A}_{i}\left[\phi \tilde{\varrho} \tilde{u}^{i}\right] \overline{T_{k}(\tilde{\varrho})} \partial_{j} \underline{\phi}-\tilde{\varrho} \tilde{u}^{i} \mathcal{A}_{i}\left[\underline{\phi} \overline{T_{k}(\tilde{\varrho})}\right] \partial_{j} \phi\right) \mathrm{d} x \mathrm{~d} s \\
& =: \tilde{\mathbb{E}} \sum_{k=1}^{7} K_{k}, \quad i=1,2,3 \text {. }
\end{aligned}
$$

where a 'bar' above a function represents the limit of the corresponding approximate sequence of functions.

Lemma 4 Let $\phi(x), \phi(x) \in C_{c}^{\infty}\left(\mathbb{R}^{3}\right)$. Then the strong convergence

$$
\mathcal{R}\left[\phi \tilde{\varrho}_{n} \tilde{u}_{n}^{j}\right] \underline{\phi} T_{k}\left(\tilde{\varrho}_{n}\right)-\phi \tilde{\varrho}_{n} \tilde{u}_{n}^{j} \mathcal{R}\left[\underline{\phi} T_{k}\left(\tilde{\varrho}_{n}\right)\right] \rightarrow \mathcal{R}\left[\phi \tilde{\varrho} \tilde{u}^{j}\right] \underline{\phi} \overline{T_{k}(\tilde{\varrho})}-\phi \tilde{\varrho} \tilde{u}^{j} \mathcal{R}\left[\underline{\phi} \overline{T_{k}(\tilde{\varrho})}\right]
$$

holds in $L^{2}\left(\tilde{\Omega} \times(0, T) ; W^{-1,2}\left(\mathbb{R}^{3}\right)\right)$ where $\mathcal{R}:=\mathcal{R}_{i j}$.

Proof See [5, Sect. 6.1] or the deterministic counterpart in [28, Eq. 7.5.23].

Now by using the weak-strong pair: (33) 1 and Lemma 4, we can pass to the limit in the crucial term $I_{6}$ to get $\tilde{\mathbb{E}} I_{6} \rightarrow \tilde{\mathbb{E}} K_{6}$.

All other terms can be treated in a similar manner as in [5, Sect. 6.1] keeping in mind that the terms involving derivatives and cutoff functions are of lower order and hence easier. In particular, we obtain the convergence $\tilde{\mathbb{E}} I_{7} \rightarrow \tilde{\mathbb{E}} K_{7}$ by observing that $\mathcal{R}=\partial_{j} \mathcal{A}_{i}$.

We have therefore shown that

$$
\begin{aligned}
\lim _{n \rightarrow 0} \tilde{\mathbb{E}} \int_{Q}\left[a \tilde{\varrho}_{n}^{\gamma}-(\lambda+2 v) \operatorname{div} \tilde{\mathbf{u}}_{n}\right] \phi \underline{\phi} T_{k}\left(\tilde{\varrho}_{n}\right) \mathrm{d} x \mathrm{~d} t \\
=\tilde{\mathbb{E}} \int_{Q}[a \tilde{p}-(\lambda+2 v) \operatorname{div} \tilde{\mathbf{u}}] \phi \underline{\phi} \overline{T_{k}(\tilde{\varrho})} \mathrm{d} x \mathrm{~d} t
\end{aligned}
$$

\subsection{Identification of the pressure limit}

Showing that indeed $\tilde{p}=\tilde{\varrho}^{\gamma}$ or equivalently that $\tilde{\varrho}_{n} \rightarrow \tilde{\varrho}$ strongly in $L^{p}(\tilde{\Omega} \times Q)$ for all $p \in[1, \gamma+\Theta)$ follows Feireisl's approach via the use of the so-called oscillation defect measure. This is a purely deterministic argument even in our stochastic settings since it relies on the renormalized continuity equation. To avoid repetition, we refer the reader to [28, Sect. 7.3.7.3] or [11]. To confirm that it indeed applies in the stochastic setting, the reader may also refer to [5, Sects. 6.2 and 6.3].

We now conclude with the following lemma which completes the proof of Theorem 1. 
Lemma $5\left[\left(\tilde{\Omega}, \tilde{\mathscr{F}},\left(\tilde{\mathscr{F}}_{t}\right)_{t \geq 0}, \tilde{\mathbb{P}}\right), \tilde{\varrho}, \tilde{\mathbf{u}}, \tilde{W}\right]$ is a finite energy weak martingale solution of $(4)$ with initial law $\Lambda$. Furthermore, (4) $)_{1}$ is satisfied in the renormalized sense.

\section{Proof of Theorem 2}

For every $\varepsilon>0$, let assume there exits a finite energy weak martingale solution of Eq. (4) given by

$$
\left[\left(\Omega^{\varepsilon}, \mathscr{F}^{\varepsilon},\left(\mathscr{F}_{t}^{\varepsilon}\right), \mathbb{P}^{\varepsilon}\right), \varrho_{\varepsilon}, \mathbf{u}_{\varepsilon}, W_{\varepsilon}\right] .
$$

Then by setting $\bar{\varrho}=1$ and $a=\frac{1}{\varepsilon^{2}}$ in (14), and applying Taylor expansion to the function $f(\varrho)=\varrho^{\gamma}$ around $\varrho=1$, we get

$$
\begin{aligned}
\mathbb{E} & {\left[\int_{\mathbb{R}^{3}}\left(\frac{\left|\mathbf{q}_{\varepsilon}(0)\right|^{2}}{2 \varrho_{\varepsilon}(0)}+H\left(\varrho_{\varepsilon}(0)\right)\right) \mathrm{d} x\right]^{p} } \\
& =\int_{L^{\gamma} \times L^{\frac{2 \gamma}{\gamma+1}}}\left\|\frac{\left|\mathbf{q}_{\varepsilon}\right|^{2}}{2 \varrho_{\varepsilon}}+\frac{\gamma z^{\gamma-2}}{2 \varepsilon^{2}}\left(\varrho_{\varepsilon}-1\right)^{2}\right\|_{L^{1}\left(\mathbb{R}^{3}\right)}^{p} \mathrm{~d} \Lambda\left(\varrho_{\varepsilon}, \mathbf{q}_{\varepsilon}\right) \leq c_{p, T}
\end{aligned}
$$

for $z \in[\varrho, 1]$ or $z \in[1, \varrho]$ and where we have used the initial law in Theorem 1. Similar to Sect. 3, we can now collect the following uniform (in $\varepsilon$ ) bounds

$$
\begin{aligned}
\varphi_{\varepsilon} & \in L^{p}\left(\Omega ; L^{\infty}\left(0, T ; L^{\min \{2, \gamma\}}\left(\mathbb{R}^{3}\right)\right)\right), \\
\nabla \mathbf{u}_{\varepsilon} & \in L^{p}\left(\Omega ; L^{2}\left(0, T ; L^{2}\left(\mathbb{R}^{3}\right)\right)\right), \\
\sqrt{\varrho_{\varepsilon}} \mathbf{u}_{\varepsilon} & \in L^{p}\left(\Omega ; L^{\infty}\left(0, T ; L^{2}\left(\mathbb{R}^{3}\right)\right)\right), \\
\varrho_{\varepsilon} \mathbf{u}_{\varepsilon} & \in L^{p}\left(\Omega ; L^{\infty}\left(0, T ; L_{\mathrm{loc}}^{\frac{2 \gamma}{\gamma+1}}\left(\mathbb{R}^{3}\right)\right)\right), \\
\varrho_{\varepsilon} \mathbf{u}_{\varepsilon} \otimes \mathbf{u}_{\varepsilon} & \in L^{p}\left(\Omega ; L^{2}\left(0, T ; L_{\mathrm{loc}}^{\frac{6 \gamma}{4 \gamma+3}}\left(\mathbb{R}^{3}\right)\right)\right),
\end{aligned}
$$

where $\varphi_{\varepsilon}:=\frac{\varrho_{\varepsilon}-1}{\varepsilon}$ and where

$$
\varrho_{\varepsilon} \rightarrow 1 \text { in } L^{p}\left(\Omega ; L^{\infty}\left(0, T ; L_{\mathrm{loc}}^{\gamma}\left(\mathbb{R}^{3}\right)\right)\right) .
$$

cf. (19) (with $\bar{\varrho}=1$ ), (23) and [4, eqn. 3.6].

\subsection{Acoustic wave equation}

Let $\Delta^{-1}$ represent the inverse of the Laplace operator on $\mathbb{R}^{3}$ and let $\mathcal{Q}=\nabla \Delta^{-1} \operatorname{div}$ and $\mathcal{P}$ be, respectively, the gradient and solenoidal parts according to Helmoltz decomposition. Then referring again to [4], we observe that by setting $\varphi_{\varepsilon}=\frac{\varrho_{\varepsilon}-1}{\varepsilon}$ and $\mathrm{Id}=\mathcal{Q}+\mathcal{P}$, we derive from equation (4):

$$
\begin{aligned}
\varepsilon \mathrm{d} \varphi_{\varepsilon}+\operatorname{div} \mathcal{Q}\left(\varrho_{\varepsilon} \mathbf{u}_{\varepsilon}\right) \mathrm{d} t & =0 \\
\varepsilon \mathcal{Q} \Phi\left(\varrho_{\varepsilon}, \varrho_{\varepsilon} \mathbf{u}_{\varepsilon}\right) \mathrm{d} W-\gamma \nabla \varphi_{\varepsilon} \mathrm{d} t & =\varepsilon \mathrm{d} \mathcal{Q}\left(\varrho_{\varepsilon} \mathbf{u}_{\varepsilon}\right)-\varepsilon \mathbf{F}_{\varepsilon} \mathrm{d} t
\end{aligned}
$$

where

$$
\mathbf{F}_{\varepsilon}=\operatorname{div} \mathcal{Q}\left(\varrho_{\varepsilon} \mathbf{u}_{\varepsilon} \otimes \mathbf{u}_{\varepsilon}\right)-v \Delta \mathcal{Q} \mathbf{u}_{\varepsilon}-(\lambda+v) \nabla \operatorname{div} \mathbf{u}_{\varepsilon}+\frac{1}{\varepsilon^{2}} \nabla\left[\varrho_{\varepsilon}^{\gamma}-1-\gamma\left(\varrho_{\varepsilon}-1\right)\right] .
$$

Now let us observe that from $(38)_{5}$ and the continuity of $\mathcal{Q}$, we have that

$$
\operatorname{div} \mathcal{Q}\left(\varrho_{\varepsilon} \mathbf{u}_{\varepsilon} \otimes \mathbf{u}_{\varepsilon}\right) \in L^{p}\left(\Omega ; L^{2}\left(0, T ; W_{\mathrm{loc}}^{-1, \frac{6 \gamma}{4 \gamma+3}}\left(\mathbb{R}^{3}\right)\right)\right)
$$


independently of $\varepsilon$. And that

$$
\nu \Delta \mathcal{Q} \mathbf{u}_{\varepsilon}+(\lambda+v) \nabla \operatorname{div} \mathbf{u}_{\varepsilon} \in L^{p}\left(\Omega ; L^{2}\left(0, T ; W_{\mathrm{loc}}^{-1,2}\left(\mathbb{R}^{3}\right)\right)\right)
$$

uniformly in $\varepsilon$ by virtue of $(38)_{2}$. Lastly, the choice of $a=\frac{1}{\varepsilon^{2}}$ and $\bar{\varrho}_{\varepsilon}=1$ in the pressure potential (11) of the energy estimate (10) and Taylor's theorem means that for $s:=\min \{2, \gamma\}>1$,

$$
\frac{1}{\varepsilon^{2}} \nabla\left[\varrho_{\varepsilon}^{\gamma}-1-\gamma\left(\varrho_{\varepsilon}-1\right)\right] \in L^{p}\left(\Omega ; L^{\infty}\left(0, T ; W_{\mathrm{loc}}^{-1, s}\left(\mathbb{R}^{3}\right)\right)\right) .
$$

uniformly in $\varepsilon$. cf. (14) for $\bar{\varrho}=1$ and (38) 1 . By combining (41), (42) and (43) with the embeddings $W^{-1, \frac{6 \gamma}{4 \gamma+3}}\left(B_{r}\right) \hookrightarrow W^{-l, 2}\left(B_{r}\right)$ and $W^{-1, s}\left(B_{r}\right) \hookrightarrow W^{-l, 2}\left(B_{r}\right)$, where $B_{r}$ is a ball of radius $r>0$, it holds that for $l>5 / 2$,

$$
\mathbf{F}_{\varepsilon} \in L^{p}\left(\Omega ; L^{2}\left(0, T ; W_{\mathrm{loc}}^{-l, 2}\left(\mathbb{R}^{3}\right)\right)\right)
$$

uniformly in $\varepsilon$.

\subsection{Compactness}

To explore compactness for the acoustic equation, let first define the path space $\chi=\chi_{\varrho} \times$ $\chi_{\mathbf{u}} \times \chi_{\varrho \mathbf{u}} \times \chi_{W}$ where

$$
\begin{array}{rlrl}
\chi_{\varrho} & =C_{\omega}\left([0, T] ; L_{\mathrm{loc}}^{\gamma}\left(\mathbb{R}^{3}\right)\right), & \chi_{\mathbf{u}}=\left(L^{2}\left(0, T ; W_{\mathrm{loc}}^{1,2}\left(\mathbb{R}^{3}\right)\right), \omega\right), \\
\chi_{\varrho \mathbf{u}}=C_{\omega}\left([0, T] ; L_{\mathrm{loc}}^{\frac{2 \gamma}{\gamma+1}}\left(\mathbb{R}^{3}\right)\right), & \chi_{W}=C\left([0, T] ; \mathfrak{U}_{0}\right),
\end{array}
$$

and let

1. $\mu_{\varrho_{\varepsilon}}$ be the law of $\varrho_{\varepsilon}$ on the space $\chi_{\varrho}$,

2. $\mu_{\mathbf{u}_{\varepsilon}}$ be the law of $\mathbf{u}_{\varepsilon}$ on $\chi_{\mathbf{u}}$,

3. $\mu_{\mathcal{P}\left(\varrho_{\varepsilon} \mathbf{u}_{\varepsilon}\right)}$ be the law of $\mathcal{P}\left(\varrho_{\varepsilon} \mathbf{u}_{\varepsilon}\right)$ on the space $\chi_{\varrho \mathbf{u}}$,

4. $\mu_{W}$ be the law of $W$ on the space $\chi_{W}$,

5. $\mu^{\varepsilon}$ be the joint law of $\varrho_{\varepsilon}, \mathbf{u}_{\varepsilon}, \mathcal{P}\left(\varrho_{\varepsilon} \mathbf{u}_{\varepsilon}\right)$, and $W$ on the space $\chi$.

Then the following lemma, the proof of which is similar to [4, Corollary 3.7], holds true.

Lemma 6 The sets $\left\{\mu^{\varepsilon} ; \varepsilon \in(0,1)\right\}$ is tight on $\chi$.

Now similarly as in Proposition 3, we apply the Jakubowski-Skorokhod representation theorem [20] to get the following proposition.

Proposition 5 There exists a subsequence $\mu^{\varepsilon}$, a probability space $(\tilde{\Omega}, \tilde{\mathscr{F}}, \tilde{\mathbb{P}})$ with $\chi$-valued Borel measurable random variables $\left(\tilde{\varrho}_{\varepsilon}, \tilde{\mathbf{u}}_{\varepsilon}, \tilde{\mathbf{q}}_{\varepsilon}, \tilde{W}_{\varepsilon}\right), n \in \mathbb{N}$, and $(\tilde{\varrho}, \tilde{\mathbf{u}}, \tilde{\mathbf{q}}, \tilde{W})$ such that

- the law of $\left(\tilde{\varrho}_{\varepsilon}, \tilde{\mathbf{u}}_{\varepsilon}, \tilde{\mathbf{q}}_{\varepsilon}, \tilde{W}_{\varepsilon}\right)$ is given by $\mu^{\varepsilon}, \varepsilon \in(0,1)$,

- the law of $(\tilde{\varrho}, \tilde{\mathbf{u}}, \tilde{\mathbf{q}}, \tilde{W})$, denoted by $\mu$ is a Randon measure,

- $\left(\tilde{\varrho}_{\varepsilon}, \tilde{\mathbf{u}}_{\varepsilon}, \tilde{\mathbf{q}} \varepsilon, \tilde{W}_{\varepsilon}\right)$ converges $\tilde{\mathbb{P}}-$ a.s to $(\tilde{\varrho}, \tilde{\mathbf{u}}, \tilde{\mathbf{q}}, \tilde{W})$ in the topology of $\chi$.

To extend this new probability space $(\tilde{\Omega}, \tilde{\mathscr{F}}, \tilde{\mathbb{P}})$ into a stochastic basis, we endow it with the $\tilde{\mathbb{P}}$-augmented canonical filtrations for $\left(\tilde{\varrho}_{\varepsilon}, \tilde{\mathbf{u}}_{\varepsilon}, \tilde{W}_{\varepsilon}\right)$ and $(\tilde{\varrho}, \tilde{\mathbf{u}}, \tilde{W})$, respectively, by setting

$$
\tilde{\mathscr{F}}_{t}^{\varepsilon}=\sigma\left(\sigma\left(\mathbf{r}_{t} \tilde{\varrho}_{\varepsilon}, \mathbf{r}_{t} \tilde{\mathbf{u}}_{\varepsilon}, \mathbf{r}_{t} \tilde{W}_{\varepsilon}\right) \cup\{N \in \tilde{\mathscr{F}} ; \tilde{\mathbb{P}}(N)=0\}\right), \quad t \in[0, T]
$$




$$
\tilde{\mathscr{F}}_{t}=\sigma\left(\sigma\left(\mathbf{r}_{t} \tilde{\mathbf{u}}, \mathbf{r}_{t} \tilde{W}\right) \cup\{N \in \tilde{\mathscr{F}} ; \tilde{\mathbb{P}}(N)=0\}\right), \quad t \in[0, T] .
$$

where $\mathbf{r}_{t}$ is the continuous function defined in (31) above adapted to the spaces defined in this section.

\subsection{Identification of the limit}

We now verify that our new processes

$$
\left[\left(\tilde{\Omega}, \tilde{\mathscr{F}},\left(\tilde{\mathscr{F}}_{t}^{\varepsilon}\right), \tilde{\mathbb{P}}\right), \tilde{\varrho}_{\varepsilon}, \tilde{\mathbf{u}}_{\varepsilon}, \tilde{W}_{\varepsilon}\right] \operatorname{and}\left[\left(\tilde{\Omega}, \tilde{\mathscr{F}},\left(\tilde{\mathscr{F}}_{t}\right), \tilde{\mathbb{P}}\right), \tilde{\mathbf{u}}, \tilde{W}\right]
$$

are indeed finite energy weak martingale solutions and a weak martingale solution, respectively, for Eqs. (4) and (5).

Proposition $6\left[\left(\tilde{\Omega}, \tilde{\mathscr{F}},\left(\tilde{\mathscr{F}}_{t}^{\varepsilon}\right)_{t \geq 0}, \tilde{\mathbb{P}}\right), \tilde{\varrho}_{\varepsilon}, \tilde{\mathbf{u}}_{\varepsilon}, \tilde{W}_{\varepsilon}\right]$ is a finite energy weak martingale solution of Eq. (4) with initial law $\Lambda_{\varepsilon}$ for $\varepsilon \in(0,1)$.

The proof of this proposition is similar to [4, Proposition 3.10].

Consequently, the uniform bounds shown in (38), (39) and (44) earlier hold for these corresponding random processes on this new space. In particular, we have that

$$
\begin{gathered}
\tilde{\varphi}_{\varepsilon} \in L^{p}\left(\Omega ; L^{\infty}\left(0, T ; L^{\min \{2, \gamma\}}\left(\mathbb{R}^{3}\right)\right)\right), \\
\tilde{\mathbf{F}}_{\varepsilon} \in L^{p}\left(\Omega ; L^{2}\left(0, T ; W_{\mathrm{loc}}^{-l, 2}\left(\mathbb{R}^{3}\right)\right)\right), \\
\tilde{\mathbf{u}}_{\varepsilon} \in L^{p}\left(\Omega ; L^{2}\left(0, T ; W_{\mathrm{loc}}^{1,2}\left(\mathbb{R}^{3}\right)\right)\right), \\
\tilde{\varrho}_{\varepsilon} \tilde{\mathbf{u}}_{\varepsilon} \in L^{p}\left(\Omega ; L^{\infty}\left(0, T ; L_{\mathrm{loc}}^{\frac{2 \gamma}{\gamma+1}}\left(\mathbb{R}^{3}\right)\right)\right)
\end{gathered}
$$

holds uniformly in $\varepsilon$ for $p \in[1, \infty)$ and where $l>5 / 2, \tilde{\varphi}_{\varepsilon}=\frac{\tilde{\varrho}_{\varepsilon}-1}{\varepsilon}$ and

$$
\tilde{\mathbf{F}}_{\varepsilon}=\operatorname{div} \mathcal{Q}\left(\tilde{\varrho}_{\varepsilon} \tilde{\mathbf{u}}_{\varepsilon} \otimes \tilde{\mathbf{u}}_{\varepsilon}\right)-v \Delta \mathcal{Q} \tilde{\mathbf{u}}_{\varepsilon}-(\lambda+v) \nabla \operatorname{div} \tilde{\mathbf{u}}_{\varepsilon}+\frac{1}{\varepsilon^{2}} \nabla\left[\tilde{\varrho}_{\varepsilon}^{\gamma}-1-\gamma\left(\tilde{\varrho}_{\varepsilon}-1\right)\right]
$$

We now verify that indeed the limit process satisfies Definition 2. This will complete the proof of Theorem 2 .

Proposition $7\left[\left(\tilde{\Omega}, \tilde{\mathscr{F}},\left(\tilde{\mathscr{F}}_{t}\right)_{t \geq 0}, \tilde{\mathbb{P}}\right), \tilde{\mathbf{u}}, \tilde{W}\right]$ is a weak martingale solution of Eq. (5) with initial law $\Lambda$.

Proof The proof of this proposition will follow from the following lemmata and propositions.

Lemma 7 For all $t \in[0, T]$ and $\phi \in C_{c}^{\infty}\left(\mathbb{R}^{3}\right)$, we let

$$
\begin{aligned}
M(\varrho, \mathbf{u}, \mathbf{q})_{t}= & \langle\mathbf{q}(t), \phi\rangle-\langle\mathbf{q}(0), \phi\rangle-\int_{0}^{t}\langle\mathbf{q} \otimes \mathbf{u}, \nabla \phi\rangle \mathrm{d} s+v \int_{0}^{t}\langle\nabla \mathbf{u}, \nabla \phi\rangle \mathrm{d} s \\
& +(\lambda+v) \int_{0}^{t}\langle\operatorname{div} \mathbf{u}, \operatorname{div} \phi\rangle \mathrm{d} s-\frac{1}{\varepsilon^{2}} \int_{0}^{t}\left\langle\varrho^{\gamma}, \operatorname{div} \phi\right\rangle \mathrm{d} s .
\end{aligned}
$$

Then $M\left(\tilde{\varrho}_{\varepsilon}, \tilde{\mathbf{u}}_{\varepsilon}, \tilde{\varrho}_{\varepsilon} \tilde{\mathbf{u}}_{\varepsilon}\right)_{t} \rightarrow M(1, \tilde{\mathbf{u}}, \tilde{\mathbf{u}})_{t} \tilde{\mathbb{P}}-$ a.s. as $\varepsilon \rightarrow 0$.

Proof The proof of this lemma follows from combining Proposition 5 with the Lemmata 8, 10 and Proposition 8 below. 
Lemma 8 For every $q<6$, the following $\tilde{\mathbb{P}}-$ a.s. convergence holds:

$$
\begin{aligned}
&\left(\tilde{\varrho}_{\varepsilon}-1\right) \rightarrow 0 \text { in } L^{\infty}\left(0, T ; L^{\min \{2, \gamma\}}\left(\mathbb{R}^{3}\right)\right), \\
& \mathcal{P}\left(\tilde{\varrho}_{\varepsilon} \tilde{\mathbf{u}}_{\varepsilon}\right) \rightarrow \tilde{\mathbf{u}} \text { in } L^{2}\left(0, T ; W_{\mathrm{loc}}^{-1,2}\left(\mathbb{R}^{3}\right)\right), \\
& \mathcal{P} \tilde{\mathbf{u}}_{\varepsilon} \rightarrow \tilde{\mathbf{u}} \text { in } L^{2}\left(0, T ; L_{\mathrm{loc}}^{q}\left(\mathbb{R}^{3}\right)\right) .
\end{aligned}
$$

Proof See [4].

Remark 8 Henceforth, we write ' $\lesssim$ ' for ' $\leq c$ ' and ' $\vec{\sim}$ ' for ' $=c$ ' where $c$ which may varies from line to line is some universal constant that is independent of $\varepsilon$.

Proposition 8 The strong convergence below holds:

$$
\mathcal{Q}\left(\tilde{\varrho}_{\varepsilon} \tilde{\mathbf{u}}_{\varepsilon}\right) \rightarrow 0 \text { in } L^{2}\left(0, T ; L_{\text {loc }}^{\frac{2 \gamma}{\gamma+1}}\left(\mathbb{R}^{3}\right)\right) \tilde{\mathbb{P}}-\text { a.s. }
$$

Proof Let define the function $\tilde{\Psi}_{\varepsilon}=\Delta^{-1} \operatorname{div}\left(\tilde{\varrho}_{\varepsilon} \tilde{\mathbf{u}}_{\varepsilon}\right)$ such that $\nabla \tilde{\Psi}_{\varepsilon}=\mathcal{Q}\left(\tilde{\varrho}_{\varepsilon} \tilde{\mathbf{u}}_{\varepsilon}\right)$. Then, equation (40) becomes:

$$
\begin{aligned}
\varepsilon \mathrm{d}\left(\tilde{\varphi}_{\varepsilon}\right)+\Delta \tilde{\Psi}_{\varepsilon} \mathrm{d} t & =0 \\
\varepsilon \mathrm{d} \nabla \tilde{\Psi}_{\varepsilon}+\gamma \nabla \tilde{\varphi}_{\varepsilon} \mathrm{d} t & =\varepsilon \tilde{\mathbf{F}}_{\varepsilon} \mathrm{d} t+\varepsilon \mathcal{Q} \Phi\left(\tilde{\varrho}_{\varepsilon}, \tilde{\varrho}_{\varepsilon} \tilde{\mathbf{u}}_{\varepsilon}\right) \mathrm{d} \tilde{W}_{\varepsilon} .
\end{aligned}
$$

We, however, observe that Eq. (49) is equivalent to

$$
\varepsilon \mathrm{d}\left[\begin{array}{c}
\tilde{\varphi}_{\varepsilon} \\
\nabla \tilde{\Psi}_{\varepsilon}
\end{array}\right]=\mathcal{A}\left[\begin{array}{c}
\tilde{\varphi}_{\varepsilon} \\
\nabla \tilde{\Psi}_{\varepsilon}
\end{array}\right] \mathrm{d} t+\varepsilon\left[\begin{array}{c}
0 \\
\tilde{\mathbf{F}}_{\varepsilon}
\end{array}\right] \mathrm{d} t+\varepsilon\left[\begin{array}{c}
0 \\
\mathcal{Q} \Phi
\end{array}\right] \mathrm{d} \tilde{W}_{\varepsilon}
$$

where the usual wave operator

$$
\mathcal{A}=\left[\begin{array}{cc}
0 & -\operatorname{div} \\
-\gamma \nabla & 0
\end{array}\right]
$$

is an infinitesimal generator of a strongly continuous semigroup $S(\cdot)=\exp (\mathcal{A} \cdot)$. See for example [8]. Also $\Phi:=\Phi(\tilde{\varrho}, \tilde{\varrho} \tilde{\mathbf{u}})$ is the Hilbert-Schmidt operator, and equation (49) is satisfied weakly in the probability sense, it follows that this weak solution is also a mild solution, see for example [7, Theorem 6.5]. As such after rescaling, we obtain the mild equation

$$
\begin{aligned}
{\left[\begin{array}{c}
\tilde{\varphi}_{\varepsilon} \\
\nabla \tilde{\Psi}_{\varepsilon}
\end{array}\right](t)=} & S\left(\frac{t}{\varepsilon}\right)\left[\begin{array}{c}
\tilde{\varphi}_{\varepsilon}(0) \\
\nabla \tilde{\Psi}_{\varepsilon}(0)
\end{array}\right]+\int_{0}^{t} S\left(\frac{t-s}{\varepsilon}\right)\left[\begin{array}{c}
0 \\
\tilde{\mathbf{F}}_{\varepsilon}
\end{array}\right] \mathrm{d} s \\
& +\int_{0}^{t} S\left(\frac{t-s}{\varepsilon}\right)\left[\begin{array}{c}
0 \\
\mathcal{Q} \tilde{\Phi}_{\varepsilon}
\end{array}\right] \mathrm{d} \tilde{W}_{s, \varepsilon}
\end{aligned}
$$

where the semigroup $S(t)$ is such that

$$
S(t)\left[\begin{array}{c}
\tilde{\varphi}_{0} \\
\nabla \tilde{\Psi}_{0}
\end{array}\right]=\left[\begin{array}{c}
\tilde{\varphi} \\
\nabla \tilde{\Psi}
\end{array}\right](t)
$$

is the solution to the homogeneous problem:

$$
\begin{aligned}
\mathrm{d}(\tilde{\varphi})+\Delta \tilde{\Psi} \mathrm{d} t & =0 \\
\mathrm{~d} \nabla \tilde{\Psi}+\gamma \nabla \tilde{\varphi} \mathrm{d} t & =0 . \\
\tilde{\varphi}(0) & =\tilde{\varphi}_{0} ; \quad \nabla \tilde{\Psi}(0)=\nabla \tilde{\Psi}_{0} .
\end{aligned}
$$


Using Fourier transforms (in space), we obtain solution of Eq. (54) which is given by the pair

$$
\begin{gathered}
\nabla \tilde{\Psi}(t, x)=\frac{e^{i \sqrt{-\gamma \Delta t}}}{2}\left(\nabla \tilde{\Psi}_{0}(x)-\frac{i \sqrt{\gamma}}{\sqrt{-\Delta}} \tilde{\varphi}_{0}(x)\right)+\frac{e^{-i \sqrt{-\gamma \Delta} t}}{2}\left(\nabla \tilde{\Psi}_{0}(x)+\frac{i \sqrt{\gamma}}{\sqrt{-\Delta}} \tilde{\varphi}_{0}(x)\right) \\
\tilde{\varphi}(t, x)=\frac{e^{i \sqrt{-\gamma \Delta t}}}{2}\left(\frac{i \sqrt{-\Delta}}{\sqrt{\gamma}} \nabla \tilde{\Psi}_{0}(x)+\tilde{\varphi}_{0}(x)\right)-\frac{e^{-i \sqrt{-\gamma \Delta t}}}{2}\left(\frac{i \sqrt{-\Delta}}{\sqrt{\gamma}} \nabla \tilde{\Psi}_{0}(x)-\tilde{\varphi}_{0}(x)\right) .
\end{gathered}
$$

The lemma below is crucial to the proof of Proposition 8 and is an adaptation of [31, Lemma 2.2] to our setting. cf. [12, Lemma 3.1].

Lemma 9 Let $\phi(x) \in C_{c}^{\infty}\left(\mathbb{R}^{3}\right)$, we have

$$
\int_{\mathbb{R}}\left\|e^{i \sqrt{-\gamma \Delta t}}[\mathbf{v} \phi]\right\|_{L^{2}\left(\mathbb{R}^{3}\right)}^{2} \mathrm{~d} t \leq c(\phi)\|\mathbf{v}\|_{L^{2}\left(\mathbb{R}^{3}\right)}^{2}
$$

for any $\mathbf{v} \in L^{2}\left(\mathbb{R}^{3}\right)$.

Proof For simplicity, we assume that $\gamma=1$. General $\gamma>1$ will then follow by rescaling $\delta$ below.

Using Plancherel's theorem in $t$ and $x$, we have that

$$
\begin{aligned}
& \int_{\mathbb{R}}\left\|e^{i \sqrt{-\Delta} t}[\mathbf{v} \phi]\right\|_{L^{2}\left(\mathbb{R}^{3}\right)}^{2} \mathrm{~d} t=c(\pi) \int_{\mathbb{R}} \int_{\mathbb{R}^{3}}\left|\int_{\mathbb{R}^{3}} \widehat{\phi}(\xi-\eta) \delta(\tau-|\eta|) \widehat{\mathbf{v}}(\eta) \mathrm{d} \eta\right|^{2} \mathrm{~d} \xi \mathrm{d} \tau \\
& =c(\pi) \int_{\mathbb{R}} \int_{\mathbb{R}^{3}}\left|\int_{\{\tau=|\eta|\}} \widehat{\phi}(\xi-\eta) \widehat{\mathbf{v}}(\eta) \mathrm{d} S_{\eta}\right|^{2} \mathrm{~d} \xi \mathrm{d} \tau \\
& \quad \leq c(\pi) \int_{\mathbb{R}} \int_{\mathbb{R}^{3}}\left(\int_{\{\tau=|\eta|\}}|\widehat{\phi}(\xi-\eta)| \mathrm{d} S_{\eta}\right)\left(\int_{\{\tau=|\eta|\}}|\widehat{\phi}(\xi-\eta)||\widehat{\mathbf{v}}(\eta)|^{2} \mathrm{~d} S_{\eta}\right) \mathrm{d} \xi \mathrm{d} \tau \\
& \quad \leq c(\pi, \phi) \int_{\mathbb{R}^{3}} \int_{\mathbb{R}} \int_{\{\tau=|\eta|\}}|\widehat{\phi}(\xi-\eta)||\widehat{\mathbf{v}}(\eta)|^{2} \mathrm{~d} S_{\eta} \mathrm{d} \tau \mathrm{d} \xi \\
& \quad \leq c(\pi, \phi) \int_{\mathbb{R}^{3}} \int_{\mathbb{R}^{3}}|\widehat{\phi}(\xi-\eta)||\widehat{\mathbf{v}}(\eta)|^{2} \mathrm{~d} \eta \mathrm{d} \xi \leq c(\pi, \phi)\|\mathbf{v}\|_{L^{2}\left(\mathbb{R}^{3}\right)}^{2}
\end{aligned}
$$

where we have used the Cauchy-Schwartz inequality.

Moving on, we now consider a smooth cut-off function (with expanding support) $\eta_{r} \in$ $C_{0}^{\infty}\left(B_{2 r}\right)$ with $\eta_{r} \equiv 1$ in $B_{r}$ for $r>0$ and zero elsewhere. We now mollify the product of this cut-off function and our functions in (49) by means of spatial convolution with the standard mollifier. That is, if $v$ is one of the functions in (49), we set

$$
v^{\kappa}=\left(\eta_{r} v\right) * \varphi^{\kappa}
$$

where $\varphi^{\kappa}$ is the standard mollifier. This we do to ensure that the regularized functions are globally integrable. First off, we note that since $(45)_{4}$ holds uniformly in $\varepsilon$, for an arbitrary small $\delta>0$, we can find a $\kappa(\delta)$ such that

$$
\tilde{\mathbb{E}} \sup _{t \in[0, T]}\left\|\left(\tilde{\varrho}_{\varepsilon} \tilde{\mathbf{u}}_{\varepsilon}\right)^{\kappa}-\tilde{\varrho}_{\varepsilon} \tilde{\mathbf{u}}_{\varepsilon}\right\|_{L^{\frac{2 \gamma}{\gamma+1}}(B)}^{p} \leq \delta
$$


for any $1 \leq p<\infty$ and an arbitrary ball $B \subset \subset B_{r}$ for $r>0$. Then by using (53), (55) and Lemma 9, we have that

$$
\tilde{\mathbb{E}}\left\|S(t)\left[\begin{array}{c}
\tilde{\varphi}_{0}^{\kappa} \\
\nabla \tilde{\Psi}_{0}^{\kappa}
\end{array}\right]\right\|_{L^{2}(\mathbb{R} \times B)}^{2} \leq c_{h, \gamma} \tilde{\mathbb{E}}\left\|\left[\begin{array}{c}
\tilde{\varphi}_{0}^{\kappa} \\
\nabla \tilde{\Psi}_{0}^{\kappa}
\end{array}\right]\right\|_{L^{2}\left(\mathbb{R}^{3}\right)}^{2}
$$

for any ball $B \subset \mathbb{R}^{3}$ and where in particular, the constant is independent of $\kappa$. So by rescaling in time, i.e, setting $s=\frac{t}{\varepsilon}$ so that $\mathrm{d} s=\frac{\mathrm{d} t}{\varepsilon}$, we get

$$
\begin{aligned}
\tilde{\mathbb{E}}\left\|S\left(\frac{t}{\varepsilon}\right)\left[\begin{array}{c}
\tilde{\varphi}_{\varepsilon}^{\kappa}(0) \\
\nabla \tilde{\Psi}_{\varepsilon}^{\kappa}(0)
\end{array}\right]\right\|_{L^{2}((0, T) \times B)}^{2} & \leq \tilde{\mathbb{E}}\left\|S\left(\frac{t}{\varepsilon}\right)\left[\begin{array}{c}
\tilde{\varphi}_{\varepsilon}^{\kappa}(0) \\
\nabla \tilde{\Psi}_{\varepsilon}^{\kappa}(0)
\end{array}\right]\right\|_{L^{2}(\mathbb{R} \times B)}^{2} \\
& \lesssim \varepsilon \tilde{\mathbb{E}}\left\|\left[\begin{array}{c}
\tilde{\varphi}_{\varepsilon}^{\kappa}(0) \\
\nabla \tilde{\Psi}_{\varepsilon}^{\kappa}(0)
\end{array}\right]\right\|_{L^{2}\left(\mathbb{R}^{3}\right)}^{2}
\end{aligned}
$$

with a constant that is independent of $\varepsilon$. Now by the continuity of $\mathcal{Q},(56)$, and the initial law defined in the statement of Theorem 1 , we conclude that

$$
\begin{aligned}
\tilde{\mathbb{E}}\left\|S\left(\frac{t}{\varepsilon}\right)\left[\begin{array}{c}
\tilde{\varphi}_{\varepsilon}^{\kappa}(0) \\
\nabla \tilde{\Psi}_{\varepsilon}^{\kappa}(0)
\end{array}\right]\right\|_{L^{2}((0, T) \times B)}^{2} & \lesssim \varepsilon \tilde{\mathbb{E}}\left(\left\|\tilde{\varphi}_{\varepsilon}^{\kappa}(0)\right\|_{L^{\min \{2, \gamma\}}\left(\mathbb{R}^{3}\right)}^{2}+\left\|\tilde{\mathbf{q}}_{\varepsilon}^{\kappa}(0)\right\|_{L^{\frac{2 \gamma}{\gamma+1}}\left(\mathbb{R}^{3}\right)}^{2}\right) \\
& \leq \varepsilon c_{\kappa, M} .
\end{aligned}
$$

Similarly we have that for any ball $B \subset \mathbb{R}^{3}$,

$$
\begin{aligned}
\tilde{\mathbb{E}}\left\|\int_{0}^{t} S\left(\frac{t-s}{\varepsilon}\right) \tilde{\mathbf{F}}_{\varepsilon}^{\kappa} \mathrm{d} s\right\|_{L^{2}((0, T) \times B)}^{2} & \leq \tilde{\mathbb{E}}\left\|S\left(\frac{t-s}{\varepsilon}\right) \tilde{\mathbf{F}}_{\varepsilon}^{\kappa}\right\|_{L^{2}((0, t) \times(0, T) \times B)}^{2} \\
& \leq \tilde{\mathbb{E}}\left\|S\left(\frac{t}{\varepsilon}\right) S\left(\frac{-s}{\varepsilon}\right) \tilde{\mathbf{F}}_{\varepsilon}^{\kappa}\right\|_{L^{2}(\mathbb{R} \times(0, T) \times B)}^{2} \\
& \leq \varepsilon c_{\gamma} \tilde{\mathbb{E}}\left\|S\left(\frac{-s}{\varepsilon}\right) \tilde{\mathbf{F}}_{\varepsilon}^{\kappa}\right\|_{L^{2}((0, T) \times B)}^{2} \\
& \sim \varepsilon \tilde{\mathbb{E}}\left\|\tilde{\mathbf{F}}_{\varepsilon}^{\kappa}\right\|_{L^{2}\left((0, T) \times \mathbb{R}^{3}\right)}^{2} \leq \varepsilon c_{\gamma, \kappa}
\end{aligned}
$$

Where we have used Jensen's inequality and Fubini's theorem in the first inequality, extended $(0, t)$ to $\mathbb{R}$ and used the semigroup property in the second inequality, applied similar reasoning as in (58) in the third inequality and then used that $(S(t))_{t}$ is a group of isometries on $L^{2}$ (extended by zero outside of the ball) in the last line above.

We have therefore obtained the following bounds

$$
\begin{aligned}
\tilde{\mathbb{E}}\left\|S\left(\frac{t}{\varepsilon}\right)\left[\begin{array}{c}
\tilde{\varphi}_{\varepsilon}^{\kappa}(0) \\
\nabla \tilde{\Psi}_{\varepsilon}^{\kappa}(0)
\end{array}\right]\right\|_{L^{2}\left(0, T ; L^{2}(B)\right)}^{2} & \lesssim \varepsilon, \quad \tilde{\mathbb{E}}\left\|\int_{0}^{t} S\left(\frac{t-s}{\varepsilon}\right)\left[\begin{array}{c}
0 \\
\tilde{\mathbf{F}}_{\varepsilon}^{\kappa}
\end{array}\right] \mathrm{d} s\right\|_{L^{2}\left(0, T ; L^{2}(B)\right)}^{2} \\
& \lesssim \varepsilon
\end{aligned}
$$

for any ball $B \subset \mathbb{R}^{3}$. Now let make the notation $\tilde{\Phi}_{\varepsilon}^{\kappa}\left(e_{i}\right):=g_{i}\left(\cdot, \tilde{\varrho}_{\varepsilon}(\cdot),\left(\tilde{\mathbf{q}}_{\varepsilon}\right)(\cdot)\right)^{\kappa}=: \tilde{g}_{i}^{\varepsilon, \kappa}$. We also notice that for a continuous function $S(t)$ and a continuous operator $\mathcal{Q}$, the quantity 
$S(t) \mathcal{Q} \Phi$ is Hilbert-Schmidt if $\Phi$ is Hilbert-Schmidt. As such, it follows from Itó isometry that

$$
\begin{aligned}
\tilde{\mathbb{E}}\left\|\int_{0}^{t} S\left(\frac{t-s}{\varepsilon}\right)\left[\begin{array}{c}
0 \\
\mathcal{Q} \tilde{\Phi}_{\varepsilon}^{\kappa}
\end{array}\right] \mathrm{d} \tilde{W}_{\varepsilon}(s)\right\|_{L^{2}((0, T) \times B)}^{2} \\
\quad=\tilde{\mathbb{E}} \int_{0}^{t}\left\|S\left(\frac{t-s}{\varepsilon}\right) \mathcal{Q} \tilde{\Phi}_{\varepsilon}^{\kappa}\right\|_{L_{2}\left(\mathfrak{U} ; L^{2}((0, T) \times B)\right)}^{2} \mathrm{~d} s \\
\quad=\tilde{\mathbb{E}} \int_{0}^{t} \sum_{i \in \mathbb{N}}\left\|S\left(\frac{t-s}{\varepsilon}\right) \mathcal{Q} \tilde{g}_{i}^{\varepsilon, \kappa}\right\|_{L^{2}((0, T) \times B)}^{2} \mathrm{~d} s \\
\quad \lesssim \int_{0}^{T} \sum_{i \in \mathbb{N}} \int_{\mathbb{R}} \tilde{\mathbb{E}}\left\|S\left(\frac{t-s}{\varepsilon}\right) \mathcal{Q} \tilde{g}_{i}^{\varepsilon, \kappa}\right\|_{L^{2}(B)}^{2} \mathrm{~d} s \mathrm{~d} t
\end{aligned}
$$

where the above involved extending $s$ from $(0, t)$ to $\mathbb{R}$ as well as Fubini's theorem.

Now using the semigroup property and similar estimate as in equation (57) and (58), followed by the fact that the semigroup is an isometry with respect to the $L^{2}$-norm, we get that

$$
\begin{aligned}
& \int_{0}^{T} \sum_{i \in \mathbb{N}} \int_{\mathbb{R}} \tilde{\mathbb{E}}\left\|S\left(\frac{t-s}{\varepsilon}\right) \mathcal{Q} \tilde{g}_{i}^{\varepsilon, \kappa}\right\|_{L^{2}(B)}^{2} \mathrm{~d} s \mathrm{~d} t \\
&=\int_{0}^{T} \sum_{i \in \mathbb{N}} \tilde{\mathbb{E}}\left\|S\left(\frac{t}{\varepsilon}\right) S\left(\frac{-s}{\varepsilon}\right) \mathcal{Q} \tilde{g}_{i}^{\varepsilon, \kappa}\right\|_{L^{2}(\mathbb{R} \times B)}^{2} \mathrm{~d} t \\
& \lesssim \varepsilon \int_{0}^{T} \sum_{i \in \mathbb{N}} \tilde{\mathbb{E}}\left\|S\left(\frac{-s}{\varepsilon}\right) \mathcal{Q} \tilde{g}_{i}^{\varepsilon, \kappa}\right\|_{L^{2}(B)}^{2} \mathrm{~d} t \\
& \approx \varepsilon \int_{0}^{T} \sum_{i \in \mathbb{N}} \tilde{\mathbb{E}}\left\|\mathcal{Q} \tilde{g}_{i}^{\varepsilon, \kappa}\right\|_{L^{2}\left(\mathbb{R}^{3}\right)}^{2} \mathrm{~d} t \lesssim \varepsilon \int_{0}^{T} \sum_{i \in \mathbb{N}} \tilde{\mathbb{E}}\left\|\tilde{g}_{i}^{\varepsilon, \kappa}\right\|_{L^{2}\left(\mathbb{R}^{3}\right)}^{2} \mathrm{~d} t \\
& \lesssim \varepsilon \varepsilon \int_{0}^{T} \sum_{i \in \mathbb{N}}\left\|\tilde{g}_{i}^{\varepsilon}\right\|_{L^{1}\left(\mathbb{R}^{3}\right)}^{2} \mathrm{~d} t \lesssim \varepsilon .
\end{aligned}
$$

The last inequality follows because the noise term is assumed to be compactly supported in $\mathbb{R}^{3}$. See (6). We have that

$$
\tilde{\mathbb{E}}\left\|\int_{0}^{t} S\left(\frac{t-s}{\varepsilon}\right) \mathcal{Q} \tilde{\Phi}_{\varepsilon}^{\kappa} \mathrm{d} \tilde{W}_{\varepsilon}(s)\right\|_{L^{2}((0, T) \times B)}^{2} \leq \varepsilon c_{h, \gamma, \kappa}
$$


where the constant is independent $\varepsilon$. Combining this with the estimates from (61), we get from (52) that

$$
\begin{aligned}
\tilde{\mathbb{E}}\left\|\left[\begin{array}{c}
\tilde{\varphi}_{\varepsilon}(t) \\
\nabla \tilde{\Psi}_{\varepsilon}(t)
\end{array}\right]\right\|_{L^{2}((0, T) \times B)}^{2} & =\tilde{\mathbb{E}}\left\|\tilde{\varphi}_{\varepsilon}(t)\right\|_{L^{2}((0, T) \times B)}^{2}+\tilde{\mathbb{E}}\left\|\nabla \tilde{\Psi}_{\varepsilon}(t)\right\|_{L^{2}((0, T) \times B)}^{2} \\
& \lesssim I_{1}+I_{2}+I_{3} \leq \varepsilon c_{h, \gamma, \kappa} .
\end{aligned}
$$

where we have set

$$
\begin{aligned}
& I_{1}:=\tilde{\mathbb{E}}\left\|S\left(\frac{t}{\varepsilon}\right)\left[\begin{array}{c}
\tilde{\varphi}_{\varepsilon}(0) \\
\nabla \tilde{\Psi}_{\varepsilon}(0)
\end{array}\right]\right\|_{L^{2}((0, T) \times B)}^{2} \\
& I_{2}:=\tilde{\mathbb{E}}\left\|\int_{0}^{t} S\left(\frac{t-s}{\varepsilon}\right) \tilde{\mathbf{F}}_{\varepsilon} \mathrm{d} s\right\|_{L^{2}((0, T) \times B)}^{2} \\
& I_{3}:=\tilde{\mathbb{E}}\left\|\int_{0}^{t} S\left(\frac{t-s}{\varepsilon}\right) \mathcal{Q} \Phi \mathrm{d} W_{s, \varepsilon}\right\|_{L^{2}((0, T) \times B)}^{2}
\end{aligned}
$$

So in particular,

$$
\tilde{\mathbb{E}}\left\|\nabla \tilde{\Psi}_{\varepsilon}^{\kappa}(t)\right\|_{L^{2}((0, T) \times B)}^{2} \leq \varepsilon c_{h, \gamma, \kappa}
$$

holds for any ball $B \subset \mathbb{R}^{3}$. We also deduce from Eq. (56) together with the embedding $L_{t}^{\infty} L_{x}^{r} \hookrightarrow L_{t}^{2} L_{x}^{r}$ where $r=\frac{2 \gamma}{\gamma+1}$, and the continuity of $\mathcal{Q}$ that

$$
\tilde{\mathbb{E}}\left\|\nabla \tilde{\Psi}_{\varepsilon}^{\kappa}-\nabla \tilde{\Psi}_{\varepsilon}\right\|_{L^{2}\left(0, T ; L^{r}(B)\right)}^{2} \leq c_{\delta, t}, \quad \tilde{\mathbb{E}}\left\|\tilde{\mathbf{q}}_{\varepsilon}^{\kappa}-\tilde{\mathbf{q}}_{\varepsilon}\right\|_{L^{2}\left(0, T ; L^{r}(B)\right)}^{2} \leq c_{\delta, t}
$$

where $\delta$ is the arbitrarily constant from (56) which is independent of $\kappa$ and $\varepsilon$. As such, the constant $c_{\delta, t}$ can be made arbitrarily small for an arbitrary choice of $\delta$ so that

$$
\lim _{\kappa \downarrow 0} \tilde{\mathbb{E}}\left\|\nabla \tilde{\Psi}_{\varepsilon}^{\kappa}-\nabla \tilde{\Psi}_{\varepsilon}\right\|_{L^{2}\left(0, T ; L^{r}(B)\right)}^{2}=0, \quad r=\frac{2 \gamma}{\gamma+1} .
$$

Thus, it follows from (62) and the uniform bound (63) that we may exchange the order of taking limits in (63). As such for any ball $B \subset \mathbb{R}^{3}$, we have that

$$
\begin{aligned}
0 & \leq \lim _{\varepsilon \downarrow 0} \tilde{\mathbb{E}}\left\|\nabla \tilde{\Psi}_{\varepsilon}\right\|_{L^{2}\left(0, T ; L^{r}(B)\right)}^{2}=\lim _{\kappa \downarrow 0} \lim _{\varepsilon \downarrow 0} \tilde{\mathbb{E}}\left\|\nabla \tilde{\Psi}_{\varepsilon}\right\|_{L^{2}\left(0, T ; L^{r}(B)\right)}^{2} \\
& \leq 2 \lim _{\varepsilon \downarrow 0} \lim _{\kappa \downarrow 0} \tilde{\mathbb{E}}\left\|\nabla \tilde{\Psi}_{\varepsilon}^{\kappa}-\nabla \tilde{\Psi}_{\varepsilon}\right\|_{L^{2}\left(0, T ; L^{r}(B)\right)}^{2}+2 \lim _{\kappa \downarrow 0} \lim _{\varepsilon \downarrow 0} \tilde{\mathbb{E}}\left\|\nabla \tilde{\Psi}_{\varepsilon}^{\kappa}\right\|_{L^{2}\left(0, T ; L^{r}(B)\right)}^{2} \\
& \leq c\left(\lim _{\kappa \downarrow 0} \tilde{\mathbb{E}}\left\|\nabla \tilde{\Psi}_{\varepsilon}^{\kappa}-\nabla \tilde{\Psi}_{\varepsilon}\right\|_{L^{2}\left(0, T ; L^{r}(B)\right)}^{2}+\lim _{\varepsilon \downarrow 0} \tilde{\mathbb{E}}\left\|\nabla \tilde{\Psi}_{\varepsilon}^{\kappa}\right\|_{L^{2}((0, T) \times B)}^{2}\right)=0
\end{aligned}
$$

hence our claim.

Remark 9 We observe that by combining (47) and Proposition 8, we can only conclude that

$$
\tilde{\varrho}_{\varepsilon} \tilde{\mathbf{u}}_{\varepsilon} \rightarrow \tilde{\mathbf{u}} \text { in } L^{2}\left(0, T ; W_{\mathrm{loc}}^{-1,2}\left(\mathbb{R}^{3}\right)\right)
$$

$\tilde{\mathbb{P}}$-a.s.

However, we can improve this spatial regularity. We give this as part of the lemma below. 
Lemma 10 Let $\gamma>\frac{3}{2}, q<6$ and $l>\frac{3}{2}$. Then for all $r \in\left(\frac{3}{2}, 6\right)$, we have that

$$
\begin{array}{r}
\operatorname{div}\left(\tilde{\varrho}_{\varepsilon} \tilde{\mathbf{u}}_{\varepsilon} \otimes \tilde{\mathbf{u}}_{\varepsilon}\right) \rightarrow \operatorname{div}(\tilde{\mathbf{u}} \otimes \tilde{\mathbf{u}}) \quad \text { in } \quad L^{1}\left(0, T ; W_{\operatorname{div}}^{-l, 2}(B)\right), \\
\tilde{\varrho}_{\varepsilon} \tilde{\mathbf{u}}_{\varepsilon} \rightarrow \tilde{\mathbf{u}} \text { in } L^{2}\left(0, T ; L^{r}(B)\right)
\end{array}
$$

$\tilde{\mathbb{P}}-$ a.s. for any ball $B \subset \mathbb{R}^{3}$.

Proof To avoid repetition, we refer the reader to [4, Proposition 3.13] for the proof of (66). However we proof (67) below.

By using the identity $\mathcal{P}\left(\tilde{\varrho}_{\varepsilon} \tilde{\mathbf{u}}_{\varepsilon}\right)=\mathcal{P}\left(\tilde{\varrho}_{\varepsilon}-1\right) \tilde{\mathbf{u}}_{\varepsilon}+\mathcal{P} \tilde{\mathbf{u}}_{\varepsilon}$, the reverse triangle inequality and then the triangle inequality, we have that

$$
\begin{aligned}
& \left|\left\|\mathcal{P}\left(\tilde{\varrho}_{\varepsilon} \tilde{\mathbf{u}}_{\varepsilon}\right)\right\|_{L^{2}\left(0, T ; L^{r}(B)\right)}-\|\tilde{\mathbf{u}}\|_{L^{2}\left(0, T ; L^{r}(B)\right)}\right| \\
& \quad \leq\left\|\mathcal{P}\left(\tilde{\varrho}_{\varepsilon}-1\right) \tilde{\mathbf{u}}_{\varepsilon}+\mathcal{P} \tilde{\mathbf{u}}_{\varepsilon}-\tilde{\mathbf{u}}\right\|_{L^{2}\left(0, T ; L^{r}(B)\right)} \\
& \quad \leq\left\|\mathcal{P}\left(\tilde{\varrho}_{\varepsilon}-1\right) \tilde{\mathbf{u}}_{\varepsilon}\right\|_{L^{2}\left(0, T ; L^{r}(B)\right)}+\left\|\mathcal{P} \tilde{\mathbf{u}}_{\varepsilon}-\tilde{\mathbf{u}}\right\|_{L^{2}\left(0, T ; L^{r}(B)\right)} \\
& \quad \leq c\left\{\left\|\tilde{\varrho}_{\varepsilon}-1\right\|_{L^{\infty}\left(0, T ; L^{\min \{2, \gamma\}}\left(\mathbb{R}^{3}\right)\right)}\left\|\tilde{\mathbf{u}}_{\varepsilon}\right\|_{L^{2}\left(0, T ; L^{\frac{r \gamma}{\gamma-r}}(B)\right)}+\left\|\mathcal{P} \tilde{\mathbf{u}}_{\varepsilon}-\tilde{\mathbf{u}}\right\|_{L^{2}\left(0, T ; L^{q}(B)\right)}\right\} \\
& \quad \rightarrow 0
\end{aligned}
$$

where we have used $(45)_{3},(46),(48)$ and the continuity of $\mathcal{P}$.

Combining this with Proposition 8 finishes the proof.

By combining (46) with Lemma 10, we finish the proof of Lemma 7.

The following lemma now completes the proof of Proposition 7.

Lemma 11 For all $t \in[0, T]$ and $\phi \in C_{c}^{\infty}\left(\mathbb{R}^{3}\right)$, we define

$$
\begin{aligned}
& N(\varrho, \mathbf{q})_{t}=\sum_{k \in \mathbb{N}} \int_{0}^{t}\left\langle g_{k}(\varrho, \mathbf{q}), \phi\right\rangle^{2} \mathrm{~d} s \\
& N_{k}(\varrho, \mathbf{q})_{t}=\int_{0}^{t}\left\langle g_{k}(\varrho, \mathbf{q}), \phi\right\rangle \mathrm{d} s .
\end{aligned}
$$

Then we have that for $\varepsilon \in(0,1)$

$$
\begin{gathered}
N\left(\tilde{\varrho}_{\varepsilon}, \tilde{\varrho}_{\varepsilon} \tilde{\mathbf{u}}_{\varepsilon}\right)_{t} \rightarrow N(1, \tilde{\mathbf{u}})_{t} \quad \tilde{\mathbb{P}}-\text { a.s. } \\
N_{k}\left(\tilde{\varrho}_{\varepsilon}, \tilde{\varrho}_{\varepsilon} \tilde{\mathbf{u}}_{\varepsilon}\right)_{t} \rightarrow N_{k}(1, \tilde{\mathbf{u}})_{t} \quad \tilde{\mathbb{P}}-\text { a.s. }
\end{gathered}
$$

as $\varepsilon \rightarrow 0$.

Proof By Minkowski's inequality, we have that

$$
\begin{aligned}
& \left\|\left\langle\Phi\left(\tilde{\varrho}_{\varepsilon}, \tilde{\varrho}_{\varepsilon} \tilde{\mathbf{u}}_{\varepsilon}\right) \cdot, \phi\right\rangle-\langle\Phi(1, \tilde{\mathbf{u}}) \cdot, \phi\rangle\right\|_{L_{2}(\mathfrak{U} ; \mathbb{R})} \\
& =\left(\sum_{k \in \mathbb{N}}\left|\left\langle\left(\Phi\left(\tilde{\varrho}_{\varepsilon}, \tilde{\varrho}_{\varepsilon} \tilde{\mathbf{u}}_{\varepsilon}\right)-\Phi(1, \tilde{\mathbf{u}})\right)\left(e_{k}\right), \phi\right\rangle\right|^{2}\right)^{\frac{1}{2}}
\end{aligned}
$$




$$
\begin{aligned}
& \leq c(\phi)\left(\sum_{k \in \mathbb{N}}\left|\int_{\operatorname{supp}(\phi)}\left(g_{k}\left(\tilde{\varrho}_{\varepsilon}, \tilde{\varrho}_{\varepsilon} \tilde{\mathbf{u}}_{\varepsilon}\right)-g_{k}(1, \tilde{\mathbf{u}})\right) \mathrm{d} x\right|^{2}\right)^{\frac{1}{2}} \\
& \leq c \int_{\operatorname{supp}(\phi)}\left(\sum_{k \in \mathbb{N}}\left|g_{k}\left(\tilde{\varrho}_{\varepsilon}, \tilde{\varrho}_{\varepsilon} \tilde{\mathbf{u}}_{\varepsilon}\right)-g_{k}(1, \tilde{\mathbf{u}})\right|^{2}\right)^{\frac{1}{2}} \mathrm{~d} x
\end{aligned}
$$

where $\int_{\operatorname{supp}(\phi)} f \mathrm{~d} x$ is the restriction of the integral of $f$ to the support of $\phi$.

Now let $\mathbf{x}:=\left(\tilde{\varrho}_{\varepsilon}, \tilde{\varrho}_{\varepsilon} \tilde{\mathbf{u}}_{\varepsilon}\right)$ and $\mathbf{y}:=(1, \tilde{\mathbf{u}})$ be vectors in $\mathbb{R}^{4}$ and define the line segment joining them by

$$
L(\mathbf{x}, \mathbf{y})=\{t \mathbf{x}+(1+t) \mathbf{y}: 0 \leq t \leq 1\}
$$

Then by the Mean value inequality, we can find $\left(\underline{\varrho}_{\varepsilon}, \underline{\mathbf{q}}_{\varepsilon}\right) \in L(\mathbf{x}, \mathbf{y})$ such that

$$
\begin{aligned}
& \int_{\operatorname{supp}(\phi)}\left(\sum_{k \in \mathbb{N}}\left|g_{k}\left(\tilde{\varrho}_{\varepsilon}, \tilde{\varrho}_{\varepsilon} \tilde{\mathbf{u}}_{\varepsilon}\right)-g_{k}(1, \tilde{\mathbf{u}})\right|^{2}\right)^{\frac{1}{2}} \mathrm{~d} x \\
& \quad \leq \int_{\operatorname{supp}(\phi)}\left(\left|\left(\tilde{\varrho}_{\varepsilon}, \tilde{\varrho}_{\varepsilon} \tilde{\mathbf{u}}_{\varepsilon}\right)-(1, \tilde{\mathbf{u}})\right|^{2} \sum_{k \in \mathbb{N}}\left|\nabla_{\underline{\varrho}_{\varepsilon}, \underline{\mathbf{q}}_{\varepsilon}} g_{k}\left(\underline{\varrho_{\varepsilon}}, \underline{\mathbf{q}_{\varepsilon}}\right)\right|^{2}\right)^{\frac{1}{2}} \mathrm{~d} x \\
& \quad \leq c\left(\int_{\operatorname{supp}(\phi)}\left|\tilde{\varrho}_{\varepsilon}-1\right| \mathrm{d} x+\int_{\operatorname{supp}(\phi)}\left|\tilde{\varrho}_{\varepsilon} \tilde{\mathbf{u}}_{\varepsilon}-\tilde{\mathbf{u}}\right| \mathrm{d} x\right) \\
& \quad=: I_{1}+I_{2}
\end{aligned}
$$

where we have used (7) and [19, Eq. 6.13.6] in the last inequality.

Hence by using the embeddings $L^{\min \{2, \gamma\}} \hookrightarrow L^{1}$ and $L^{r} \hookrightarrow L^{1}$, which holds true for any compact set or ball in $\mathbb{R}^{3}$ and where $r$ is as defined in Lemma 10, we get that $I_{1} \rightarrow 0$ and $I_{2} \rightarrow 0$ for a.e. $(\omega, t)$ in $\tilde{\Omega} \times(0, T)$. This is due to (46) and (67). Hence

$$
\left\langle\Phi\left(\tilde{\varrho}_{\varepsilon}, \tilde{\varrho}_{\varepsilon} \tilde{\mathbf{u}}_{\varepsilon}\right) \cdot, \phi\right\rangle \rightarrow\langle\Phi(1, \tilde{\mathbf{u}}) \cdot, \phi\rangle \text { in } L_{2}(\mathfrak{U} ; \mathbb{R}) \quad \tilde{\mathbb{P}} \times \mathcal{L}-\text { a.e. }
$$

which implies that

$$
N\left(\tilde{\varrho}_{\varepsilon}, \tilde{\varrho}_{\varepsilon} \tilde{\mathbf{u}}_{\varepsilon}\right)_{t} \rightarrow N(1, \tilde{\mathbf{u}})_{t} \quad \text { in } \quad L_{2}(\mathfrak{U} ; \mathbb{R}) \quad \tilde{\mathbb{P}} \times \mathcal{L}-\text { a.e. }
$$

Similar argument holds for $N_{k}\left(\tilde{\varrho}_{\varepsilon}, \tilde{\varrho}_{\varepsilon} \tilde{\mathbf{u}}_{\varepsilon}\right)_{t} \rightarrow N_{k}(1, \tilde{\mathbf{u}})_{t} \quad \tilde{\mathbb{P}}-$ a.s.

Using Lemmata 7 and 11, we can now pass to the limit in equation [4, Eqs. 3.14-3.16] to get that :

$$
\begin{aligned}
\tilde{\mathbb{E}} h\left(\mathbf{r}_{s} \tilde{\mathbf{u}}, \mathbf{r}_{s} \tilde{W}\right)\left[M(1, \tilde{\mathbf{u}}, \tilde{\mathbf{u}})_{s, t}\right] & =0, \\
\tilde{\mathbb{E}} h\left(\mathbf{r}_{s} \tilde{\mathbf{u}}, \mathbf{r}_{s} \tilde{W}\right)\left[\left[M(1, \tilde{\mathbf{u}}, \tilde{\mathbf{u}})^{2}\right]_{s, t}-N(1, \tilde{\mathbf{u}})_{s, t}\right] & =0, \\
\tilde{\mathbb{E}} h\left(\mathbf{r}_{s} \tilde{\mathbf{u}}, \mathbf{r}_{s} \tilde{W}\right)\left[\left[M(1, \tilde{\mathbf{u}}, \tilde{\mathbf{u}}) \tilde{\beta}_{k}\right]_{s, t}-N(1, \tilde{\mathbf{u}})_{s, t}\right] & =0 .
\end{aligned}
$$

Equation $(68)$ means that $M(1, \tilde{\mathbf{u}}, \tilde{\mathbf{u}})_{t}$ is a $\left(\mathscr{F}_{t}\right)$-martingale. Moreover, using $(68)_{2}$, we get the quadratic and cross-variation of $M(1, \tilde{\mathbf{u}}, \tilde{\mathbf{u}})_{t}$ as:

$$
\begin{aligned}
\left\langle\left\langle M(1, \tilde{\mathbf{u}}, \tilde{\mathbf{u}})_{t}\right\rangle\right. & =N(1, \tilde{\mathbf{u}}) \\
\left\langle\left\langle M(1, \tilde{\mathbf{u}}, \tilde{\mathbf{u}})_{t}, \tilde{\beta}_{k}\right\rangle\right\rangle & =N_{k}(1, \tilde{\mathbf{u}})
\end{aligned}
$$


which yields

$$
\left\langle\left\langle M(1, \tilde{\mathbf{u}}, \tilde{\mathbf{u}})_{t}-\int_{0}^{t}\langle\Phi(1, \tilde{\mathbf{u}}) \mathrm{d} \tilde{W}, \phi\rangle\right\rangle\right\rangle=0 .
$$

That is, for $\phi \in C_{c, \operatorname{div}}^{\infty}\left(\mathbb{R}^{3}\right)$ and $t \in[0, T]$, we have that

$$
\langle\tilde{\mathbf{u}}(t), \phi\rangle=\langle\tilde{\mathbf{u}}(0), \phi\rangle+\int_{0}^{t}\langle\tilde{\mathbf{u}} \otimes \tilde{\mathbf{u}}, \nabla \phi\rangle \mathrm{d} s-v \int_{0}^{t}\langle\nabla \tilde{\mathbf{u}}, \nabla \phi\rangle \mathrm{d} s+\int_{0}^{t}\langle\Phi(1, \tilde{\mathbf{u}}) \mathrm{d} \tilde{W}, \phi\rangle
$$

$\tilde{\mathbb{P}}$-a.s. keeping in mind that $\operatorname{div} \phi=0$.

Acknowledgements The author would like to acknowledge the financial support of the Department of Mathematics, Heriot-Watt University, through the James-Watt scholarship. He will also like to thank D. Breit for recommending this work and for his many usual discussions.

Open Access This article is distributed under the terms of the Creative Commons Attribution 4.0 International License (http://creativecommons.org/licenses/by/4.0/), which permits unrestricted use, distribution, and reproduction in any medium, provided you give appropriate credit to the original author(s) and the source, provide a link to the Creative Commons license, and indicate if changes were made.

\section{References}

1. Bensoussan, A., Temam, R.: Équations stochastiques du type Navier-Stokes. J. Funct. Anal. 13, 195-222 (1973)

2. Borchers, W., Sohr, H.: On the equations rot $v=g$ and div $u=f$ with zero boundary conditions. Hokkaido Math. J. 19(1), 67-87 (1990). doi:10.14492/hokmj/1381517172

3. Breit, D., Feireisl, E., Hofmanová, M.: Compressible fluids driven by stochastic forcing: the relative energy inequality and applications. Commun. Math. Phys. 350(2), 443-473 (2015). doi:10.1007/s00220017-2833-X

4. Breit, D., Feireisl, E., Hofmanová, M.: Incompressible limit for compressible fluids with stochastic forcing. Arch. Ration. Mech. Anal. 222(2), 895-926 (2016). doi:10.1007/s00205-016-1014-y

5. Breit, D., Hofmanová, M.: Stochastic Navier-Stokes equations for compressible fluids. Indiana Univ. Math. J. 65(4), 1183-1250 (2016)

6. Capinski, M., Gatarek, D.: Stochastic equations in Hilbert space with application to Navier-Stokes equations in any dimension. J. Funct. Anal. 126(1), 26-35 (1994)

7. Da Prato, G., Zabczyk, J.: Stochastic Equations in Infinite Dimensions, vol. 152. Cambridge University Press, Cambridge (2014)

8. Desjardins, B., Grenier, E.: Low mach number limit of viscous compressible flows in the whole space. In: Proceedings of the Royal Society of London A: Mathematical, Physical and Engineering Sciences, vol. 455, pp. 2271-2279. The Royal Society (1999)

9. Diening, L., Ruzicka, M., Schumacher, K.: A decomposition technique for John domains. Ann. Acad. Sci. Fenn. Math. 35(1), 87-114 (2010)

10. Farwig, R., Sohr, H.: Generalized resolvent estimates for the Stokes system in bounded and unbounded domains. J. Math. Soc. Jpn. 46(4), 607-643 (1994). doi:10.2969/jmsj/04640607

11. Feireisl, E.: On compactness of solutions to the compressible isentropic Navier-Stokes equations when the density is not square integrable. Comment. Math. Univ. Carol. 42(1), 83-98 (2001)

12. Feireisl, E., Gallagher, I., Gerard-Varet, D., Novotný, A.: Multi-scale analysis of compressible viscous and rotating fluids. Comm. Math. Phys. 314(3), 641-670 (2012). doi:10.1007/s00220-012-1533-9

13. Feireisl, E., Maslowski, B., Novotný, A.: Compressible fluid flows driven by stochastic forcing. J. Differ. Equ. 254(3), 1342-1358 (2013). doi:10.1016/j.jde.2012.10.020

14. Feireisl, E., Novotný, A.: Singular Limits in Thermodynamics of Viscous Fluids. Springer Science \& Business Media, Berlin (2009)

15. Feireisl, E., Novotný, A., Petzeltová, H.: On the existence of globally defined weak solutions to the Navier-Stokes equations. J. Math. Fluid Mech. 3(4), 358-392 (2001)

16. Flandoli, F., Gatarek, D.: Martingale and stationary solutions for stochastic Navier-Stokes equations. Probab. Theory Relat. Fields 102(3), 367-391 (1995) 
17. Galdi, G.P.: An Introduction to the Mathematical Theory of the Navier-Stokes Equations. Steady-State Problems. Springer Monographs in Mathematics, 2nd edn. Springer, New York (2011). doi:10.1007/9780-387-09620-9

18. Geißert, M., Heck, H., Hieber, M.: On the equation div $\mathrm{u}=\mathrm{g}$ and bogovskiis operator in sobolev spaces of negative order. In: Koelink, E., van Neerven, J., de Pagter, B., Sweers, G., Luger, A., Woracek, H. (eds.) Partial Differential Equations and Functional Analysis. Operator Theory: Advances and Applications, vol. 168, pp. 113-121. Birkhäuser, Basel (2006). doi:10.1007/3-7643-7601-5_7

19. Hardy, G.H., Littlewood, J.E., Pólya, G.: Inequalities. Cambridge University Press, Cambridge (1952)

20. Jakubowski, A.: Short communication: the almost sure skorokhod representation for subsequences in nonmetric spaces. Theory Probab. Appl. 42(1), 167-174 (1998)

21. Klainerman, S., Majda, A.: Singular limits of quasilinear hyperbolic systems with large parameters and the incompressible limit of compressible fluids. Commun. Pure Appl. Math. 34(4), 481-524 (1981). doi:10. 1002/cpa.3160340405

22. Leray, J.: Sur le mouvement d'un liquide visqueux emplissant l'espace. Acta Math. 63(1), 193-248 (1934). doi:10.1007/BF02547354

23. Lions, P.L.: Mathematical topics in fluid mechanics. Vol. 2. Compressible models. Oxford Lecture Series in Mathematics and its Applications, vol. 10. The Clarendon Press, Oxford University Press, New York (1998)

24. Lions, P.L., Masmoudi, N.: Incompressible limit for a viscous compressible fluid. J. Math. Pures Appl. (9) 77(6), 585-627 (1998). doi:10.1016/S0021-7824(98)80139-6

25. Lions, P.L., Masmoudi, N.: Unicité des solutions faibles de Navier-Stokes dans $L^{N}(\Omega)$. C. R. Acad. Sci. Paris Sér. I Math. 327(5), 491-496 (1998). doi:10.1016/S0764-4442(99)80028-7

26. Lions, P.L., Masmoudi, N.: Une approche locale de la limite incompressible. C. R. Acad. Sci. Paris Sér. I Math. 329(5), 387-392 (1999). doi:10.1016/S0764-4442(00)88611-5

27. Mikulevicius, R., Rozovskii, B.L.: Global $L_{2}$-solutions of stochastic Navier-Stokes equations. Ann. Probab. 33(1), 137-176 (2005). doi:10.1214/009117904000000630

28. Novotný, A., Straškraba, I.: Introduction to the Mathematical Theory of Compressible Flow. Oxford University Press, New York (2004)

29. Robinson, J.C., Rodrigo, J.L., Sadowski, W., Vidal-López, A. (eds.): Recent progress in the theory of the Euler and Navier-Stokes equations, London Mathematical Society Lecture Note Series, vol. 430. Including paper from the workshop "The Navier-Stokes Equations in Venice" held in Venice, April 8-12, 2013. Cambridge University Press, Cambridge (2016). doi:10.1017/CBO9781316407103

30. Romito, M.: Some probabilistic topics in the Navier-Stokes equations. In: Robinson, J.C., Rodrigo, J.L., Sadowski, W., Vidal-López, A. (eds.) Recent Progress in the Theory of the Euler and NavierStokes Equations, London Mathematical Society Lecture Note Series, vol. 430, pp. 175-232. Cambridge University Press, Cambridge (2016)

31. Smith, H.F., Sogge, C.D.: Global Strichartz estimates for nontrapping perturbations of the Laplacian. Commun. Partial Differ. Equ. 25(11-12), 2171-2183 (2000). doi:10.1080/03605300008821581

32. Smith, S.: Random Perturbations of Viscous Compressible Fluids: Global Existence of Weak Solutions. arXiv preprint arXiv:1504.00951 (2015)

33. Tornatore, E.: Global solution of bi-dimensional stochastic equation for a viscous gas. NoDEA Nonlinear Differ. Equ. Appl. 7(4), 343-360 (2000). doi:10.1007/PL00001429

34. Tornatore, E., Fujita Yashima, H.: One-dimensional stochastic equations for a viscous barotropic gas. Ric. Mat. 46(2), 255-283 (1997)

35. Vaĭgant, V.A., Kazhikhov, A.V.: On the existence of global solutions of two-dimensional Navier-Stokes equations of a compressible viscous fluid. Dokl. Akad. Nauk 357(4), 445-448 (1997) 